\title{
Consistency and population sensitivity properties in marriage and roommate markets
}

\author{
Burak Can · Bettina Klaus
}

Received: 7 June 2012 / Accepted: 16 October 2012 / Published online: 29 November 2012

(C) Springer-Verlag Berlin Heidelberg 2012

\begin{abstract}
We consider one-to-one matching markets in which agents can either be matched as pairs or remain single. In these so-called roommate markets agents are consumers and resources at the same time. Klaus (Games Econ Behav 72:172-186, 2011) introduced two new "population sensitivity" properties that capture the effect newcomers have on incumbent agents: competition sensitivity and resource sensitivity. On various roommate market domains (marriage markets, no-odd-rings roommate markets, solvable roommate markets), we characterize the core using either of the population sensitivity properties in addition to weak unanimity and consistency. On the domain of all roommate markets, we obtain two associated impossibility results.
\end{abstract}

\section{Introduction}

We consider one-to-one matching markets in which agents can either be matched as pairs or remain single. These markets are known as roommate markets and they include, as special cases, the well-known marriage markets (Gale and Shapley 1962; Roth and Sotomayor 1990). Furthermore, a roommate market is a simple example of hedonic coalition as well as network formation: in a "roommate coalition" situation, only coalitions of size one or two can be formed and in a "roommate network" situation, each agent is allowed or able to form only one link (for surveys and current

B. Can

Department of Economics, Maastricht University, P.O. Box 616, 6200 MD Maastricht, The Netherlands e-mail: b.can@maastrichtuniversity.nl

B. Klaus $(\varangle)$

Faculty of Business and Economics, University of Lausanne, Internef 538, 1015 Lausanne, Switzerland e-mail: bettina.klaus@unil.ch 
research of coalition and network formation see Demange and Wooders 2004; Jackson 2008).

In these markets the commodities to be traded are the agents themselves and agents are consumers and resources at the same time. Two new "population sensitivity" properties, introduced in Klaus (2011), that capture the effect newcomers have on incumbent agents are competition and resource sensitivity: competition sensitivity requires that some incumbents will suffer if competition is caused because newcomers initiate new trades and resource sensitivity requires that some incumbents will benefit if the extra resources are consumed. The corresponding weak population sensitivity properties only consider situations when newcomers join one by one.

Both population sensitivity properties are closely related to population monotonicity, a solidarity property that requires that additional agents affect the incumbents in a similar way (either all incumbents are weakly better off or all incumbents are weakly worse off). Because of the polarization of interests that occurs in marriage markets, two specific versions of population monotonicity exist: own-side and other-side population monotonicity (Toda 2006, introduced the first and Klaus 2011, the second of these specifications). ${ }^{1}$ Klaus (2011) shows that in marriage markets, essentially own-side population monotonicity implies weak competition sensitivity and otherside population monotonicity implies weak resource sensitivity. Furthermore, Klaus (2011) presents the first characterizations of the core for solvable roommate markets using weak unanimity, ${ }^{2}$ Maskin monotonicity, ${ }^{3}$ and either weak competition or weak resource sensitivity for marriage markets and solvable roommate markets and two associated impossibility results on the domain of all roommate markets. These characterizations can be seen as corresponding results for roommate markets to one of Toda's (2006, Theorem 3.1) core characterizations for marriage markets by weak unanimity, Maskin monotonicity, and own-side population monotonicity.

In a second characterization of the core for marriage markets, Toda's (2006, Theorem 3.2) uses consistency ${ }^{4}$ instead of Maskin monotonicity. In this paper, we show how Toda's "consistency results" can be extended to roommate markets. As main results, we obtain new characterizations of the core on the domains of marriage markets, no-odd-rings roommate markets, and solvable roommate markets: on any of these domains, a solution $\varphi$ satisfies weak unanimity, consistency, and either of the population sensitivity properties if and only if it equals the core (Theorems 3 and 4). Two associated impossibility results on the domain of all roommate markets are also established (Lemmas 6(d) and 7(d)). Our results imply two corresponding "population monotonicity" results for marriage markets (Corollary 1): a solution $\varphi$ satisfies weak

\footnotetext{
1 Own-side population monotonicity: if additional men (women) enter the market, then all incumbent men (women) are weakly worse off.

Other-side population monotonicity: if additional men (women) enter the market, then all incumbent women (men) are weakly better off.

2 Weak unanimity: if a complete unanimously best matching exists, then it is chosen.

3 Maskin monotonicity: if a matching is chosen in one market, then it is also chosen in a market that results from a Maskin monotonic transformation (which essentially means that the matching improved in the ranking of all agents).

4 Consistency: if a set of matched agents leaves, then the solution should still match the remaining agents as before.
} 
unanimity, consistency, and either own-side or other-side population monotonicity if and only if it equals the core (the characterization using own-side population monotonicity is the one obtained by Toda's (2006, Theorem 3.2)). Apart from establishing new core characterizations for marriage and roommate markets (as well as some impossibilities), we obtain new insights into the working of one-sided-markets: to extend Toda's (2006, Theorem 3.2) core characterization from marriage to roommate markets, we not only had to use one of the new population sensitivity properties, we also had to develop a new proof strategy because the original two-sided market proof could not be adapted (Example 1 shows this). Another aspect of our results is the validation of population sensitivity properties as fundamental core properties. We discuss these issues in more detail in our conclusion (Sect. 5).

Our paper is organized as follows. In Sect. 2 we present the roommate model and basic properties of solutions. In Sect. 3, we introduce the variable population properties consistency, (weak) competition sensitivity, and (weak) resource sensitivity. Section 4 contains the main results. Section 5 concludes by discussing the importance of our results for the study of one-sided markets.

\section{Roommate markets}

\subsection{The model}

We consider Gale and Shapley's (1962, Example 3) roommate markets with variable sets of agents, e.g., because the allocation of dormitory rooms at a university occurs every year for different sets of students.

Let $\mathbb{N}$ be the set of potential agents. ${ }^{5}$ For a non-empty finite subset $N \subsetneq \mathbb{N}, L(N)$ denotes the set of all linear orders over $N .{ }^{6}$ For $i \in N$, we interpret $R_{i} \in L(N)$ as agent $i$ 's strict preferences over sharing a room with any of the agents in $N \backslash\{i\}$ and having a room for himself (or consuming his best outside option); e.g., j $P_{i} k P_{i} i P_{i} l$ means that $i$ would first like to share a room with $j$, then with $k$, and then $i$ would prefer to stay alone rather than sharing the room with $l$. If $j P_{i} i$, then agent $i$ finds agent $j$ acceptable and if $i P_{i} j$, then agent $i$ finds agent $j$ unacceptable. $\mathcal{R}^{N}=\prod_{N} L(N)$ denotes the set of all preference profiles of agents in $N$ (over agents in $N$ ). A roommate market consists of a finite set of agents $N \subsetneq \mathbb{N}$ and their preferences $R \in \mathcal{R}^{N}$ and is denoted by $(N, R)$. A marriage market (Gale and Shapley 1962) is a roommate market $(N, R)$ such that $N$ is the union of two disjoint sets $M$ and $W$ and each agent in $M$ (respectively $W$ ) prefers being single to being matched with any other agent in $M$ (respectively $W$ ).

A matching $\mu$ for roommate market $(N, R)$ is a function $\mu: N \rightarrow N$ of order two, i.e., for all $i \in N, \mu(\mu(i))=i$. Thus, at any matching $\mu$, the set of agents is partitioned

\footnotetext{
${ }^{5}$ Most results remain valid for a finite set of potential agents. We will explain throughout the article, which results depend on the set of potential agents to be infinite.

${ }^{6}$ A linear order over $N$ is a binary relation $\bar{R}$ that satisfies antisymmetry (for all $i, j \in N$, if $i \bar{R} j$ and $j \bar{R} i$, then $i=j$ ), transitivity (for all $i, j, k \in N$, if $i \bar{R} j$ and $j \bar{R} k$, then $i \bar{R} k$ ), and comparability (for all $i, j \in N, i \bar{R} j$ or $j \bar{R} i$ ). By $\bar{P}$ we denote the asymmetric part of $\bar{R}$. Hence, given $i, j \in N, i \bar{P} j$ means that $i$ is strictly preferred to $j ; i \bar{R} j$ means that $i \bar{P} j$ or $i=j$ and that $i$ is weakly preferred to $j$.
} 
into pairs of agents who share a room and singletons (agents who do not share a room). Agent $\mu(i)$ is agent $i$ 's match and if $\mu(i)=i$ then $i$ is matched to himself or single. For notational convenience, we often denote a matching in terms of the induced partition, e.g., for $N=\{1,2,3,4,5\}$ and matching $\mu$ such that $\mu(1)=2, \mu(3)=3$ and $\mu(4)=5$ we write $\mu=\{(1,2), 3,(4,5)\}$. For $S \subseteq N$, we denote by $\mu(S)$ the set of agents that are matched to agents in $S$, i.e., $\mu(S)=\left\{i \in N \mid \mu^{-1}(i) \in S\right\}$. We denote the set of all matchings for roommate market $(N, R)$ by $\mathcal{M}(N, R)$ (even though this set does not depend on preferences $R$ ). If it is clear which roommate market $(N, R)$ we refer to, matchings are assumed to be elements of $\mathcal{M}(N, R)$. Since agents only care about their own matches, we use the same notation for preferences over agents and matchings: for all agents $i \in N$ and matchings $\mu, \mu^{\prime}, \mu R_{i} \mu^{\prime}$ if and only if $\mu(i) R_{i} \mu^{\prime}(i)$.

Given a roommate market $(N, R)$ and $N^{\prime} \subseteq N$, we define the reduced preferences $R^{\prime} \in \mathcal{R}^{N^{\prime}}$ of $R$ to $N^{\prime}$ as follows:

(i) for all $i \in N^{\prime}, R_{i}^{\prime} \in L\left(N^{\prime}\right)$ and

(ii) for all $j, k, l \in N^{\prime}, j R_{l}^{\prime} k$ if and only if $j R_{l} k$.

We also denote the reduced preferences of $R$ to $N^{\prime}$ by $R_{N^{\prime}}$.

Given a roommate market $(N, R)$, a matching $\mu \in \mathcal{M}(N, R)$, and $N^{\prime} \subseteq N$ such that $\mu\left(N^{\prime}\right)=N^{\prime}$, the reduced (roommate) market of $(N, R)$ at $\mu$ to $N^{\prime}$ equals $\left(N^{\prime}, R_{N^{\prime}}\right)$.

Given a roommate market $(N, R)$, a matching $\mu \in \mathcal{M}(N, R)$, and $N^{\prime} \subseteq N$ such that $\mu\left(N^{\prime}\right)=N^{\prime}$, we define the reduced matching $\mu^{\prime}$ of $\mu$ to $N^{\prime}$ as follows:

(i) $\mu^{\prime}: N^{\prime} \rightarrow N^{\prime}$ and

(ii) for all $i \in N^{\prime}, \mu^{\prime}(i)=\mu(i)$.

We also denote the reduced matching of $\mu$ to $N^{\prime}$ by $\mu_{N^{\prime}}$. Note that $\mu_{N^{\prime}} \in$ $\mathcal{M}\left(N^{\prime}, R_{N^{\prime}}\right)$.

In the sequel, we consider various domains of roommate problems: the domain of all roommate markets $\mathfrak{D}$, the domain of marriage markets $\mathfrak{D}_{M}$, and later the domains of solvable and of no-odd-rings roommate markets. To avoid notational complexity when introducing solutions and their properties, we use the domain of all roommate markets $\mathfrak{D}$ with the understanding that any other domain could be used as well.

A solution $\varphi$ on $\mathfrak{D}$ is a correspondence that associates with each roommate market $(N, R) \in \mathfrak{D}$ a nonempty subset of matchings, i.e., for all $(N, R) \in \mathfrak{D}, \varphi(N, R) \subseteq$ $\mathcal{M}(N, R)$ and $\varphi(N, R) \neq \emptyset$. A subsolution $\psi$ of $\varphi$ on $\mathfrak{D}$ is a correspondence that associates with each roommate market $(N, R) \in \mathfrak{D}$ a nonempty subset of matchings in $\varphi(N, R)$, i.e., for all roommate markets $(N, R) \in \mathfrak{D}, \psi(N, R) \subseteq \varphi(N, R)$ and $\psi(N, R) \neq \emptyset$. A proper subsolution $\psi$ of $\varphi$ on $\mathfrak{D}$ is a subsolution of $\varphi$ on $\mathfrak{D}$ such that $\psi \neq \varphi$.

\subsection{Basic properties and the core}

We first introduce a voluntary participation condition based on the idea that no agent can be forced to share a room.

Individual Rationality: Let $(N, R) \in \mathfrak{D}$ and $\mu \in \mathcal{M}(N, R)$. Then, $\mu$ is individually rational if for all $i \in N, \mu(i) R_{i} i$. $I R(N, R)$ denotes the set of all these 
matchings. A solution $\varphi$ on $\mathfrak{D}$ is individually rational if it only assigns individually rational matchings, i.e., for all $(N, R) \in \mathfrak{D}, \varphi(N, R) \subseteq I R(N, R)$.

Remark 1 (Individual Rationality and (Classical) Marriage Markets)

An individually rational matching for a marriage market $(N, R) \in \mathfrak{D}_{M}$ respects the partition of agents into two types and never matches two men or two women. Hence, we embed marriage markets into our roommate market framework by an assumption on preferences (same gender agents are unacceptable) and individual rationality to ensure that no two agents of the same gender are matched. We refer to a marriage market for which matching agents of the same gender is simply assumed to not be feasible as a classical marriage market.

Next, we introduce the well-known condition of Pareto optimality and the weaker conditions of unanimity and weak unanimity.

Pareto Optimality: Let $(N, R) \in \mathfrak{D}$ and $\mu \in \mathcal{M}(N, R)$. Then, $\mu$ is Pareto optimal if there is no other matching $\mu^{\prime} \in \mathcal{M}(N, R)$ such that for all $i \in N, \mu^{\prime} R_{i} \mu$ and for some $j \in N, \mu^{\prime} P_{j} \mu . P O(N, R)$ denotes the set of all these matchings. A solution $\varphi$ on $\mathfrak{D}$ is Pareto optimal if it only assigns Pareto optimal matchings, i.e., for all $(N, R) \in \mathfrak{D}$, $\varphi(N, R) \subseteq P O(N, R)$.

(Weak) Unanimity: Let $(N, R) \in \mathfrak{D}$ and $\mu \in \mathcal{M}(N, R)$ be such that for all $i, j \in N$, $\mu(i) R_{i} j$. Then, $\mu$ is the unanimously best matching for $(N, R)$. If $\mu$ is complete, ${ }^{7}$ then, $\mu$ is the unanimously best complete matching for $(N, R)$. A solution $\varphi$ on $\mathfrak{D}$ is unanimous if it assigns the unanimously best matching whenever it exists, i.e., for all roommate markets $(N, R) \in \mathfrak{D}$ with a unanimously best matching $\mu, \varphi(N, R)=\{\mu\}$. A solution $\varphi$ on $\mathfrak{D}$ is weakly unanimous if it assigns the unanimously best complete matching whenever it exists, i.e., for all roommate markets $(N, R) \in \mathfrak{D}$ with a unanimously best complete matching $\mu, \varphi(N, R)=\{\mu\}$.

Pareto optimality implies unanimity and unanimity implies weak unanimity.

The next property requires that two agents who are "mutually best agents" are always matched with each other.

Mutually Best: Let $(N, R) \in \mathfrak{D}$ and $i, j \in N$ [possibly $i=j$ ] such that for all $k \in N$, $i R_{j} k$ and $j R_{i} k$. Then, $i$ and $j$ are mutually best agents for $(N, R)$. A matching is a mutually best matching if all mutually best agents are mutually matched. $M B(N, R)$ denotes the set of all these matchings. A solution $\varphi$ on $\mathfrak{D}$ is mutually best if it only assigns matchings at which all mutually best agents are matched, i.e., for all roommate markets $(N, R) \in \mathfrak{D}, \varphi(N, R) \subseteq M B(N, R)$.

Our notion of mutually best is slightly stronger than that used in Toda's (2006) (because he considers mutually best man-woman pairs, he does not allow for a single mutually best agent $i=j$ ). Furthermore, mutually best implies (weak) unanimity, and Pareto optimality and mutually best are logically unrelated.

\footnotetext{
7 A matching is complete if it partitions the set of agents into pairs, i.e., it contains no singletons.
} 
The above properties can also be used to define solutions, the most prominent one being the Pareto solution $P O$ that assign to each roommate market the set of Pareto optimal matchings.

Next, we define stability for roommate markets. A matching $\mu$ for roommate market $(N, R) \in \mathfrak{D}$ is blocked by a pair $\{i, j\} \subseteq N$ [possibly $i=j$ ] if $j P_{i} \mu(i)$ and $i P_{j} \mu(j)$. If $\{i, j\}$ blocks $\mu$, then $\{i, j\}$ is called a blocking pair for $\mu$. Hence, a matching $\mu$ for roommate market $(N, R) \in \mathfrak{D}$ is individually rational if there is no blocking pair $\{i, j\}$ with $i=j$ for $\mu$.

Stability, Solvability, and the Domain of Solvable Roommate Markets: Let $(N, R) \in \mathfrak{D}$ and $\mu \in \mathcal{M}(N, R)$. Then, $\mu$ is stable if there is no blocking pair for $\mu$. $S(N, R)$ denotes the set of all these matchings. A roommate market is solvable if stable matchings exist, i.e., $(N, R)$ is solvable if and only if the set of stable matchings $S(N, R) \neq \emptyset$. The domain of solvable roommate markets is denoted by $\mathfrak{D}_{S}$. Furthermore, on the domain of solvable roommate markets $\mathfrak{D}_{S}$, a solution $\varphi$ is stable if it only assigns stable matchings, i.e., for all $(N, R) \in \mathfrak{D}_{S}, \varphi(N, R) \subseteq S(N, R)$.

Gale and Shapley (1962) showed that all marriage markets are solvable, i.e., $\mathfrak{D}_{M} \subseteq \mathfrak{D}_{S}$, and they gave an example of an unsolvable roommate market (Gale and Shapley 1962, Example 3).

For many of our results we need the solvability of roommate markets and their reduced markets (Remark 3 in Sect. 3.1 explains the reason for this assumption); e.g., the domain of marriage markets is such a domain of roommate markets because it is closed with respect to the reduction operator, i.e., starting from a marriage market $(N, R) \in \mathfrak{D}_{M}$, any reduced market $\left(N^{\prime}, R_{N^{\prime}}\right)$ of $(N, R)$ is a marriage market.

Chung (2000) introduced a sufficient condition for solvability that also applies to the larger domain of weak preferences. We formulate his well-known no-odd-rings condition for our strict preference setup and refer to it as the no-odd-rings condition. ${ }^{8}$

Odd Rings and the Domain of No Odd Rings Roommate Markets: Let $(N, R) \in \mathfrak{D}$. Then, a ring for roommate market $(N, R)$ is an ordered subset of agents $\left\{i_{1}, i_{2}, \ldots, i_{k}\right\}$ $\subseteq N, k \geq 3$, such that for all $t \in\{1,2, \ldots, k\}, i_{t+1} P_{i_{t}} i_{t-1} P_{i_{t}} i_{t}$ (subscript modulo $k)$. If $k$ is odd, then $\left\{i_{1}, i_{2}, \ldots, i_{k}\right\}$ is an odd ring for roommate market $(N, R)$. A roommate market $(N, R) \in \mathfrak{D}$ is a no-odd-rings roommate market if there exists no odd ring for roommate market $(N, R)$. The domain of all such roommate markets is called the domain of no-odd-rings roommate markets and denoted by $\mathfrak{D}_{N O R}$. Note that $\mathfrak{D}_{M} \subsetneq \mathfrak{D}_{N O R} \subsetneq \mathfrak{D}_{S}$.

Another well-known concept for matching problems is the core.

Core: A matching is in the (strict or strong) core if no coalition of agents can improve their welfare by rematching among themselves. For roommate market $(N, R) \in \mathfrak{D}, \operatorname{core}(N, R)=\left\{\mu \in \mathcal{M}(N, R) \mid\right.$ there exists no $S \subseteq N$ and no $\mu^{\prime} \in$

\footnotetext{
8 Among the no-odd-rings domains listed by Chung (2000) are the Beckerian domain, single-peaked domains, single-dipped domains, and preference domains that are based on agents' representability in a metric space with the assumption that any agent prefers a match that is closer to a match that is further away.
} 
$\mathcal{M}(N, R)$ such that $\mu^{\prime}(S)=S$, for all $i \in S, \mu^{\prime}(i) R_{i} \mu(i)$, and for some $j \in S$, $\left.\mu^{\prime}(j) P_{j} \mu(j)\right\}$.

Remark 2 (Stability and the Core)

Similarly as in other matching models (e.g., marriage markets and college admissions markets), the core equals the set of stable matchings, i.e., for all $(N, R) \in \mathfrak{D}$, $\operatorname{core}(N, R)=S(N, R)$. Hence, the core is a solution on the domain of solvable roommate markets $\mathfrak{D}_{S}$ and all its subdomains, but not on the domain of all roommate markets $\mathfrak{D}$.

It is well-known that the core satisfies all properties introduced in this subsection.

Proposition 1 On the domain of solvable roommate markets (and on any of its subdomains), the core satisfies individual rationality, Pareto optimality, (weak) unanimity, mutually best, and stability.

\section{Variable population properties}

In this section we introduce and analyze properties that concern population changes.

\subsection{Consistency}

Consistency is one of the key properties in many frameworks with variable sets of agents. Thomson (2009) provides an extensive survey of consistency for various economic models, including marriage markets. For roommate markets, consistency essentially requires that when a set of matched agents leaves, then the solution should still match the remaining agents as before.

Consistency: A solution $\varphi$ on $\mathfrak{D}$ is consistent if the following holds. For each $(N, R) \in \mathfrak{D}$, each $N^{\prime} \subseteq N$, and each $\mu \in \varphi(N, R)$, if $\left(N^{\prime}, R_{N^{\prime}}\right) \in \mathfrak{D}$ is a reduced market of $(N, R)$ at $\mu$ to $N^{\prime}$ (i.e., $\left.\mu\left(N^{\prime}\right)=N^{\prime}\right)$, then $\mu_{N^{\prime}} \in \varphi\left(N^{\prime}, R_{N^{\prime}}\right)$.

For solutions defined on $\mathfrak{D}^{\prime} \subseteq \mathfrak{D}$, consistency only applies to reduced markets $\left(N^{\prime}, R_{N^{\prime}}\right) \in \mathfrak{D}^{\prime}$. Of the four domains $\left(\mathfrak{D}_{M}, \mathfrak{D}_{N O R}, \mathfrak{D}_{S}, \mathfrak{D}\right)$ that we consider, only three are closed with respect to the reduction operator, i.e., for $\mathfrak{D}^{\prime} \in\left\{\mathfrak{D}_{M}, \mathfrak{D}_{N O R}, \mathfrak{D}\right\}$, if $(N, R) \in \mathfrak{D}^{\prime}, \mu \in \mathcal{M}(N, R)$, and $\left(N^{\prime}, R_{N^{\prime}}\right)$ is a reduced market of $(N, R)$ at $\mu$, then $\left(N^{\prime}, R_{N^{\prime}}\right) \in \mathfrak{D}^{\prime}$. For the domain of solvable roommate markets $\mathfrak{D}_{S}$, non-solvable reduced markets exist and therefore consistency "looses some of its bite" (because it makes no predictions whenever market reduction leads to unsolvable reduced markets).

Remark 3 (Solvability when Studying the Core and Domain Restrictions)

Since stable matchings need not exist for the general domain of all roommate markets, we have to restrict attention to subdomains of solvable roommate markets when studying the core. Considering the whole domain of solvable roommate markets when studying consistency is difficult because a solvable roommate market might well have unsolvable reduced markets. Requiring that a solution only selects matchings that 
guarantee the solvability of all restricted markets, would already steer results forcefully towards the core. However, two domains of roommate markets we consider, $\mathfrak{D}_{M}$ and $\mathfrak{D}_{N O R}$, satisfy "closedness and solvability under the reduction operation", i.e., for any roommate market in $\mathfrak{D}^{\prime} \in\left\{\mathfrak{D}_{M}, \mathfrak{D}_{N O R}\right\}$, all possible reduced markets are (i) elements of the domain $\mathfrak{D}^{\prime}$ and (ii) solvable.

Proposition 2 On the domain of solvable roommate markets (and on any of its subdomains), the core satisfies consistency.

Proof Let $\mathfrak{D}^{\prime}$ be a (sub)domain of solvable roommate markets. Let $(N, R) \in \mathfrak{D}^{\prime}$, $\mu \in \operatorname{core}(N, R)$ and assume that $\left(N^{\prime}, R_{N^{\prime}}\right) \in \mathfrak{D}^{\prime}$ is a reduced market of $(N, R)$ at $\mu$ to $N^{\prime}$. Thus, $\operatorname{core}\left(N^{\prime}, R_{N}^{\prime}\right) \neq \varnothing$.

Assume that the core is not consistent and $\mu_{N^{\prime}} \notin \operatorname{core}\left(N^{\prime}, R_{N^{\prime}}\right)$. Hence, there exists a blocking pair $\{i, j\} \subseteq N^{\prime}$ for $\mu_{N^{\prime}}$, i.e., $j P_{i} \mu_{N^{\prime}}(i)$ and $i P_{j} \mu_{N^{\prime}}(j)$. However, since $\mu_{N^{\prime}}(i)=\mu(i)$ and $\mu_{N^{\prime}}(j)=\mu(j),\{i, j\} \subseteq N$ is also a blocking pair for $\mu$; contradicting $\mu \in \operatorname{core}(N, R)$.

\section{Lemma 1}

(a) On the domain of marriage markets (see also Toda's 2006, Lemma 3.6),

(b) On the domain of no-odd-rings roommate markets,

(c) On the domain of solvable roommate markets, no proper subsolution of the core satisfies consistency.

(d) On the domain of all roommate markets, no solution is a subsolution of the core for solvable problems and satisfies consistency (Özkal-Sanver 2010, Proposition 4.3).

We prove Lemma 1(a), (b), and (c) in Appendix A.1.

\section{Lemma 2}

(a) On the domain of marriage markets,

(b) On the domain of no-odd-rings roommate markets,

(c) On the domain of solvable roommate markets,

(d) On the domain of all roommate markets, mutually best and consistency imply individual rationality.

Proof Let $\varphi$ be a solution on any of the domains $\mathfrak{D}^{\prime}$ of Lemma 2 that satisfies mutually best and consistency. Assume, by contradiction, that there exists a roommate market $(N, R) \in \mathfrak{D}^{\prime}$, a matching $\mu \in \varphi(N, R)$, and an agent $i \in N$ such that $i P_{i} \mu(i)$. Hence, $\mu(i) \neq i$.

Let $N^{\prime}=\{i, \mu(i)\}$ and consider the reduced market $\left(N^{\prime}, R_{N^{\prime}}\right) \in \mathfrak{D}^{\prime}$ of $\mu$ to $N^{\prime}$. By consistency, $\mu_{N^{\prime}} \in \varphi\left(N^{\prime}, R^{\prime}\right)$. However, at $\left(N^{\prime}, R_{N^{\prime}}\right)$ agent $i$ is mutually best with himself and by mutually best, $\mu_{N^{\prime}} \notin \varphi\left(N^{\prime}, R^{\prime}\right)$; a contradiction.

Note that the proof of Lemma 2 does not contain any steps that are sensitive with respect to domain restrictions (except that all two-agent restricted markets used in the proof should be included in the subdomain that is considered). 


\subsection{Population sensitivity properties}

The following two population sensitivity properties were introduced and analyzed by Klaus (2011).

Consider the change of a roommate market $(N, R)$ when a finite set of agents or newcomers $\hat{N} \subsetneq \mathbb{N} \backslash N$ shows up. Then, the new set of agents is $N^{\prime}=N \cup \hat{N}$ and $\left(N^{\prime}, R^{\prime}\right), R^{\prime} \in \mathcal{R}^{N^{\prime}}$, is an extension of $(N, R)$ if $R_{N}^{\prime}=R$.

Adding a set of newcomers $\hat{N}$ might be a positive or a negative change for any of the incumbents in $N$ because it might mean

a negative change with more competition or

a positive change with more resources.

First, with competition sensitivity we formulate a property that captures the possible negative effect newcomers might have on some agents. Essentially, competition sensitivity requires that if two incumbents are newly matched after a set of newcomers arrived, then one of them suffers from the increased competition by the newcomers and is worse off.

(Weak) Competition Sensitivity (Klaus 2011): A solution $\varphi$ on $\mathfrak{D}$ is competition sensitive if the following holds. Let $(N, R) \in \mathfrak{D}$ be a roommate market and assume that $\left(N^{\prime}, R^{\prime}\right) \in \mathfrak{D}, N^{\prime}=N \cup \hat{N}$, is an extension of $(N, R)$. Then, for all $\mu \in \varphi(N, R)$ there exists $\mu^{\prime} \in \varphi\left(N^{\prime}, R^{\prime}\right)$ such that for all $i, j \in N$ [possibly $i=j$ ] that are newly matched at $\mu^{\prime}$, at least one is worse off, i.e., if $i, j \in N, \mu(i) \neq j$, and $\mu^{\prime}(i)=j$, then $\mu(i) P_{i}^{\prime} \mu^{\prime}(i)$ or $\mu(j) P_{j}^{\prime} \mu^{\prime}(j) .{ }^{9}$ A solution $\varphi$ on $\mathfrak{D}$ is weakly competition sensitive if we require competition sensitivity only when adding one newcomer at a time, i.e., $\hat{N}=\{n\}$. Note that the competition sensitivity property defined in Klaus (2008, Definition 9) equals our weak competition sensitivity.

Klaus (2011, Lemma $3^{\prime}$ ) shows that on the domains of marriage markets, solvable roommate markets, and all roommate markets, weak unanimity and weak competition sensitivity imply mutually best. We list these results below and add a corresponding result for the subdomain of no-odd-rings roommate markets.

\section{Lemma 3}

(a) On the domain of marriage markets (Klaus 2011, Lemma 3'(a)),

(b) On the domain of no-odd-rings roommate markets,

(c) On the domain of solvable roommate markets (Klaus 2011, Lemma 3'(b)),

(d) On the domain of all roommate markets (Klaus 2011, Lemma 3'(c)), weak unanimity and weak competition sensitivity imply mutually best.

The proof of Lemma 3(b) is very similar to the proof of Lemma 3(a) for marriage markets because starting from a no-odd-rings market [marriage market] one can add the newcomers in the proof such that the resulting markets are again no-odd-rings markets [marriage markets].

\footnotetext{
${ }^{9}$ Equivalently, if agents in $\hat{N}$ are leaving: for all $i, j \in N$ [possibly $i=j$ ] that are not mutually matched at $\mu$ anymore, at least one is better off, i.e., if $i, j \in N, \mu^{\prime}(i)=j$, and $\mu(i) \neq j$, then $\mu(i) P_{i} \mu^{\prime}(i)$ or $\mu(j) P_{j} \mu^{\prime}(j)$.
} 
On the domain of marriage markets, Toda's (2006, Lemma 3.1) proves that weak unanimity and own-side population monotonicity imply mutually best. The proof of Lemma 3 follows similar arguments as Toda's (2006, Lemma 3.1) proof for the corresponding marriage market result.

Example 2 in Appendix A.2 illustrates why Lemma 3 might not hold if the set of potential agents is finite.

Second, with resource sensitivity we formulate a property that captures the possible positive effect newcomers might have on some agents. Essentially, resource sensitivity requires that if two incumbents are unmatched after a set of newcomers arrived, then one of them benefits from the increase of resources by the newcomers and is better off

(Weak) Resource Sensitivity (Klaus 2011): A solution $\varphi$ on $\mathfrak{D}$ is resource sensitive if the following holds. Let $(N, R) \in \mathfrak{D}$ be a roommate market and assume that $\left(N^{\prime}, R^{\prime}\right) \in \mathfrak{D}, N^{\prime}=N \cup \hat{N}$, is an extension of $(N, R)$. Then, for all $\mu^{\prime} \in \varphi\left(N^{\prime}, R^{\prime}\right)$ there exists $\mu \in \varphi(N, R)$ such that for all $i, j \in N$ [possibly $i=j$ ] that are not mutually matched at $\mu^{\prime}$ anymore, at least one is better off, i.e., if $i, j \in N, \mu(i)=j$, and $\mu^{\prime}(i) \neq j$, then $\mu^{\prime}(i) P_{i}^{\prime} \mu(i)$ or $\mu^{\prime}(j) P_{j}^{\prime} \mu(j) .{ }^{10}$ A solution $\varphi$ on $\mathfrak{D}$ is weakly resource sensitive if we require resource sensitivity only when adding one newcomer at a time, i.e., $\hat{N}=\{n\}$. Note that the resource sensitivity property defined in Klaus (2008, Definition 11) equals our weak resource sensitivity.

Klaus (2011, Lemma 4'(a) and (c)) shows that on the domains of marriage markets and all roommate markets, weak unanimity and weak resource sensitivity imply mutually best. We list these results below and add a corresponding result for the subdomain of no-odd-rings roommate markets. Furthermore, Klaus (2011, Lemma 4(b)) shows that on the domain of solvable roommate markets, weak unanimity and resource sensitivity imply mutually best. Here, we establish the new result that on the domain of solvable roommate markets, weak unanimity, weak resource sensitivity, and consistency, imply mutually best.

\section{Lemma 4}

(a) On the domain of marriage markets (Klaus 2011, Lemma 4'(a)),

(b) On the domain of no-odd-rings roommate markets,

(c) On the domain of solvable roommate markets, consistency,

(d) On the domain of all roommate markets (Klaus 2011, Lemma 4'(c)) weak unanimity and weak resource sensitivity imply mutually best.

Proof The proof of (b) is very similar to the proof of Lemma 4(a) for marriage markets because starting from a no-odd-rings market [marriage market] one can add the newcomers in the proof such that the resulting markets are again no-odd-rings markets [marriage markets].

Proof of $(c)$ Let $\varphi$ be a solution on the domain of solvable roommate markets that satisfies consistency, weak unanimity, and weak resource sensitivity, but not mutually best.

\footnotetext{
10 Equivalently, if agents in $\hat{N}$ are leaving: for all $i, j \in N$ [possibly $i=j$ ] that are newly matched at $\mu$ at least one is worse off, i.e., if $i, j \in N, \mu^{\prime}(i) \neq j$, and $\mu(i)=j$, then $\mu^{\prime}(i) P_{i} \mu(i)$ or $\mu^{\prime}(j) P_{j} \mu(j)$.
} 
Thus, there exists a solvable roommate market $(N, R)$ and a matching $\mu \in \varphi(N, R)$ such that agents $i$ and $j$ [possibly $i=j$ ] are mutually best and $\mu(i) \neq j$. Let $\tilde{N}=\{i, j, \mu(i), \mu(j)\}$ and consider the reduced market $\left(\tilde{N}, R_{\tilde{N}}\right)$. By consistency, $\mu_{\tilde{N}} \in \varphi\left(\tilde{N}, R_{\tilde{N}}\right), i$ and $j$ are mutually best agents, and $\mu_{\tilde{N}}(i) \neq j$.

Let $\bar{N}=\{i, j\}$ and consider the reduced preferences $\bar{R}=R_{\bar{N}}$. If $i \neq j$, then there exists a unanimously best complete matching $\bar{v}$ for solvable roommate market $(\bar{N}, \bar{R}): \bar{v}$ matches agent $i$ with agent $j$. Hence, by weak unanimity, $\varphi(\bar{N}, \bar{R})=\{\bar{v}\}$ and $\bar{v}(i)=j$. If $i=j$, then $\varphi(\bar{N}, \bar{R})=\{\bar{v}\}$ and $\bar{v}(i)=j$ because $\bar{v}$ is the only possible matching. In the sequel we will not use the single-valuedness of $\varphi(\bar{N}, \bar{R})$ but that for all $\mu^{\prime} \in \varphi(\bar{N}, \bar{R}), \mu^{\prime}(i)=j$.

If $\mu(i) \neq i$, then consider the extension $\left(N^{1}, R^{1}\right)$ of $(\bar{N}, \bar{R})$ that is obtained by adding newcomer $\mu(i)$ such that $N^{1}=\bar{N} \cup\{\mu(i)\}$ and $R^{1}=R_{N^{1}} \cdot{ }^{11}$ By weak resource sensitivity, for all $\mu^{1} \in \varphi\left(N^{1}, R^{1}\right)$, there exists $\mu^{\prime} \in \varphi(\bar{N}, \bar{R})$ such that if agents $i$ and $j$ (possibly $i=j$ ) are not mutually matched at $\mu^{1}$ anymore, then at least one is better off. Then, since for all $\mu^{\prime} \in \varphi(\bar{N}, \bar{R})$ agents $i$ and $j$ are already mutually best matched, for all $\mu^{1} \in \varphi\left(N^{1}, R^{1}\right), \mu^{1}(i)=j$.

If $\mu(j) \neq j$, then we add newcomer $\mu(j)$ in a similar fashion. So we end up with the reduced market $\left(\tilde{N}, R_{\tilde{N}}\right)$. By weak resource-sensitivity, for all $\mu^{2} \in \varphi\left(\tilde{N}, R_{\tilde{N}}\right)$, $\mu^{2}(i)=j$, contradicting $\mu_{\tilde{N}}(i) \neq j$.

Lemma 4(c) cannot be established without the addition of consistency: Klaus (2011, Example 4) provides a solution on the domain of solvable roommate markets that satisfies weak unanimity and weak resource sensitivity, but neither mutually best nor consistency.

The following result slightly generalizes Klaus (2011, Proposition 2) (the proof is insensitive with respect to the specific domain of solvable roommate markets used).

Proposition 3 On the domain of solvable roommate markets (and on any of its subdomains), any stable solution satisfies competition and resource sensitivity. In particular, the core satisfies competition and resource sensitivity.

\subsection{Previous results for marriage markets}

We are aware of two papers that analyze consistency for the domain of classical marriage markets for which matching agents of the same gender is not feasible. First, Sasaki and Toda (1992) use the property together with Pareto optimality, anonymity, ${ }^{12}$ and converse consistency ${ }^{13}$ to characterize the core. (Özkal-Sanver, 2010, Proposition 4.2) shows that on the domain of all roommate markets, no solution satisfies Pareto optimality, anonymity, and converse consistency. Second, Toda's (2006) shows that

\footnotetext{
11 Note that $\left(N^{1}, R^{1}\right)$ has a unique core allocation that matches agent $i$ with agent $j$, and agent $\mu(i)$ is single.

12 Anonymity: matchings assigned by the solution do not depend on agents' names.

13 Converse consistency: matchings assigned by the solution are (conversely) related to the matchings the solution assigns to certain restricted roommate markets (with at most four agents).
} 
the core is characterized by weak unanimity, own-side population monotonicity, ${ }^{14}$ and consistency.

Theorem 1 (Toda's 2006, Theorem 3.2) On the domain of classical marriage markets, a solution satisfies weak unanimity, own-side population monotonicity, and consistency if and only if it equals the core.

Here we focus on Toda's characterization and analyze if and how the result extends from (classical) marriage markets to roommate markets. Before doing so, we obtain a new result by replacing own-side population monotonicity with other-side population monotonicity ${ }^{15}$ in Theorem 1.

Theorem 2 On the domain of classical marriage markets, a solution satisfies weak unanimity, other-side population monotonicity, and consistency if and only if it equals the core.

We prove Theorem 2 in Appendix B.

The proofs of Theorems 1 and ?? both rely on the following lemma.

Lemma 5 (Toda's 2006, Lemma 3.4) On the domain of classical marriage markets, if a solution satisfies mutually best and consistency, then it is a subsolution of the core.

We show, in Sect. 4, that Lemma 5 cannot be extended to the domain of solvable roommate markets; Example 1 in Sect. 4 shows that there exists a solution satisfying mutually best and consistency, but which assigns unstable matchings to some solvable roommate markets.

\section{Main results}

In this section we first explore some logical relations between the properties and the core. This analysis yields "subsolution of the core" results on the domains of marriage markets, no-odd-rings roommate markets, and solvable roommate markets (parts (a), (b), and (c) in Lemmas 6 and 7) and we establish two impossibility results on the domain of all roommate markets (parts (d) in Lemmas 6 and 7). Second, we establish various characterizations of the core (Theorems 3 and 4). Third, we derive two marriage market results using population monotonicity (Corollary 1 ).

\section{1 "Subsolution of the core" and impossibility results}

\section{Lemma 6}

(a) On the domain of marriage markets,

(b) On the domain of no-odd-rings roommate markets,

14 Own-side population monotonicity: if additional men (women) enter the market, then all incumbent men (women) are weakly worse off (for a formal definition see Appendix B). In Klaus (2011) we argue that the proper extension of Toda's (2006) own-side population monotonicity to roommate markets is competition sensitivity.

15 Other-side population monotonicity: if additional men (women) enter the market, then all incumbent women (men) are weakly better off (for a formal definition see Appendix B). In Klaus (2011) we argue that the proper extension of other-side population monotonicity to roommate markets is resource sensitivity. 
(c) On the domain of solvable roommate markets,

if a solution satisfies weak unanimity, competition sensitivity, and consistency, then it is a subsolution of the core.

(d) On the domain of all roommate markets, no solution satisfies weak unanimity, competition sensitivity, and consistency.

Proof Let $\varphi$ be a solution on any of the domains of Lemma 6 that satisfies weak unanimity, competition sensitivity, and consistency. By Lemma 3, $\varphi$ is mutually best and by Lemma $2, \varphi$ is individually rational.

To prove (a), (b), and (c) let $(N, R)$ be a solvable roommate market [marriage / no-odd-rings roommate market] such that $\varphi(N, R) \nsubseteq \operatorname{core}(N, R)$. To prove (d), let $(N, R)$ be an unsolvable roommate market. In all cases there exists a matching $\mu \in \varphi(N, R)$ with a blocking pair $\{i, j\}$ [possibly $i=j$ ] for $\mu$. By individual rationality, $i \neq j$.

Without loss of generality assume that $N \backslash\{i, j\}=\{1,2, \ldots, l\}$. Let $\hat{N}=$ $\left\{k_{1}, k_{2}, \ldots, k_{l}\right\} \subsetneq \mathbb{N} \backslash N$ be a set of newcomers and assume that $\left(N^{\prime}, R^{\prime}\right), N^{\prime}=N \cup \hat{N}$, is an extension of $(N, R)$ such that for all agents $m \in N \backslash\{i, j\}, m$ and $k_{m}$ are mutually best pairs and agent $m$ is the only one that finds $k_{m}$ acceptable and $k_{m}$ finds only $m$ acceptable [if $(N, R)$ is a marriage / no-odd-rings roommate market, then the newcomers and preferences can be chosen such that $\left(N^{\prime}, R^{\prime}\right)$ is also a marriage / no-odd-rings roommate market]. By mutually best, for all $\mu^{\prime} \in \varphi\left(N^{\prime}, R^{\prime}\right)$ and for all $m \in N \backslash\{i, j\}$, $\mu^{\prime}(m)=k_{m}$. By competition sensitivity, for $\mu \in \varphi(N, R)$ there exists $\hat{\mu}^{\prime} \in \varphi\left(N^{\prime}, R^{\prime}\right)$ such that agents $i$ and $j$ are not mutually matched, i.e., $\hat{\mu}^{\prime}(i) \neq j$ (if not, then agents $i$ and $j$ are newly matched at $\hat{\mu}^{\prime}$, but both are better off). Hence, $\hat{\mu}^{\prime} \in \varphi\left(N^{\prime}, R^{\prime}\right)$ is the matching that mutually best matches all agents in $N^{\prime} \backslash\{i, j\}$ and agents $i$ and $j$ are single.

Thus, $\left(\{i, j\}, R_{\{i, j\}}\right)$ is a reduced market of $\left(N^{\prime}, R^{\prime}\right)$ at $\hat{\mu}^{\prime}$ to $\{i, j\}$. Note that $i$ and $j$ are mutually best agents at $\left(\{i, j\}, R_{\{i, j\}}\right)$ and both single at $\hat{\mu}_{\{i, j\}}^{\prime}$. By consistency, $\hat{\mu}_{\{i, j\}}^{\prime} \in \varphi\left(\{i, j\}, R_{\{i, j\}}\right)$, which contradicts mutually best.

In Appendix A.2 we establish a stronger version of Lemma 6(a) and (b)Lemma 6'-using weak competition sensitivity. Whether we can strengthen Lemma 6(c) by using weak competition sensitivity instead of competition sensitivity is an open problem.

With Example 2 in Appendix A. 2 we illustrate why Lemmas 6 and $6^{\prime}$ might not hold if the set of potential agents is finite.

\section{Lemma 7}

(a) On the domain of marriage markets,

(b) On the domain of no-odd-rings roommate markets,

(c) On the domain of solvable roommate markets, if a solution satisfies weak unanimity, resource sensitivity, and consistency, then it is a subsolution of the core.

(d) On the domain of all roommate markets, no solution satisfies weak unanimity, resource sensitivity, and consistency. 
Table 1 The roommate market $(\hat{N}, \hat{R})$.

\begin{tabular}{ll}
\hline $2 \hat{P}_{1} 3 \hat{P}_{1} 4 \hat{P}_{1} 1$ & $\hat{\mu}=\{(1,3),(2,4)\}$ \\
$3 \hat{P}_{2} 4 \hat{P}_{2} 1 \hat{P}_{2} 2$ & $\hat{\mu}^{\prime}=\{(1,2),(3,4)\}$ \\
$4 \hat{P}_{3} 1 \hat{P}_{3} 2 \hat{P}_{3} 3$ & $\hat{\mu}^{\prime \prime}=\{(1,4),(2,3)\}$ \\
$1 \hat{P}_{4} 2 \hat{P}_{4} 3 \hat{P}_{4}$ & $\operatorname{core}(\hat{N}, \hat{R})=\{\hat{\mu}\}$ \\
\hline
\end{tabular}

Table 2 A separable submarket of $(N, R)$.

$2 P_{1} 3 P_{1} 4 P_{1} 1 P_{1} \ldots$

$3 P_{2} 4 P_{2} 1 P_{2} 2 P_{2} \ldots$

$4 P_{3} 1 P_{3} 2 P_{3} 3 P_{3} \ldots$

$1 P_{4} 2 P_{4} 3 P_{4} 4 P_{4} \ldots$

Proof Let $\varphi$ be a solution on any of the domains of Lemma 7 that satisfies weak unanimity, resource sensitivity, and consistency. By Lemma $4, \varphi$ is mutually best and by Lemma 2, $\varphi$ is individually rational.

To prove (a), (b), and (c) let $(N, R)$ be a solvable roommate market [marriage / no-odd-rings roommate market] such that $\varphi(N, R) \nsubseteq \operatorname{core}(N, R)$. To prove (d), let $(N, R)$ be an unsolvable roommate market. In both cases there exists a matching $\mu \in \varphi(N, R)$ with a blocking pair $\{i, j\}$ [possibly $i=j$ ] for $\mu$. By individual rationality, $i \neq j$.

Without loss of generality assume that $N \backslash\{i, j\}=\{1,2, \ldots, l\}$ and consider the roommate market $\left(\{i, j\}, R_{\{i, j\}}\right)$. There exists a unanimously best complete matching $\bar{\mu}$ for (marriage, no-odd-rings, solvable) roommate market $\left(\{i, j\}, R_{\{i, j\}}\right): \bar{\mu}$ matches agent $i$ with agent $j$. Hence, by weak unanimity, $\varphi\left(\{i, j\}, R_{\{i, j\}}\right)=\{\bar{\mu}\}$ and $\bar{\mu}(i)=$ $j$. Consider the extension $(N, R)$ of $\left(\{i, j\}, R_{\{i, j\}}\right)$ that is obtained by adding newcomers $\hat{N}=\{1, \ldots, l\}$. Because $\mu(i) \neq j$ and $\bar{\mu}(i)=j$, by resource sensitivity, $\mu(i) P_{i} \bar{\mu}(i)=j$ or $\mu(j) P_{j} \bar{\mu}(j)=i$. This contradicts that $\{i, j\}$ is a blocking pair for $\mu$.

In Appendix A. 2 we establish a stronger version of Lemma 7(a) and (b) - Lemma 7' using weak resource sensitivity.

The following solution demonstrates that corresponding results to Lemmas 5 and $7^{\prime}$ do not exist for solvable roommate markets.

Example 1 We define solution $\hat{\varphi}$ on $\mathfrak{D}^{\prime} \subseteq \mathfrak{D}_{S}$ using the following roommate market and matchings. Let $(\hat{N}, \hat{R})$ be such that $\overline{\hat{N}}=\{1,2,3,4\}$ and preferences $\hat{R}$ are given in Table 1.

The unique stable matching $\hat{\mu}$ for $(\hat{N}, \hat{R})$ matches agents 1 and 3 and agents 2 and 4. Removing any of the agents creates a "roommate cycle" for the remaining agents and the restricted roommate market is not solvable. Thus, the solvable roommate market $(\hat{N}, \hat{R})$ cannot be reached from another solvable roommate market by adding one newcomer.

If $(N, R)$ is a roommate market such that $\hat{N} \subseteq N$ and preferences $R$ are given in Table 2, then we say that $(N, R)$ is a roommate market with the separable submarket $(\hat{N}, \hat{R})$ (note that agents in $\hat{N}$ find only agents in $\hat{N}$ acceptable and any individually rational matching will match agents in $\hat{N}$ among each others). 
We now define $\hat{\varphi}$ as follows. Let $(N, R)$ be a solvable roommate market. Whenever, $(\hat{N}, \hat{R})$ is a separable submarket of $(N, R), \hat{\varphi}$ first assigns all stable matchings. Furthermore, for each stable matching $\mu$ (which matches all agents in $\hat{N}$ according to the restricted matching $\left.\mu_{\hat{N}}=\hat{\mu}\right), \hat{\varphi}$ also assigns the two matchings $\mu^{\prime}$ and $\mu^{\prime \prime}$ that correspond to $\hat{\mu}^{\prime}$ and $\hat{\mu}^{\prime \prime}$, i.e., $\mu^{\prime}\left[\mu^{\prime \prime}\right]$ matches all agents in $\hat{N}$ according to $\hat{\mu}^{\prime}\left[\hat{\mu}^{\prime \prime}\right]$ and all agents in $N \backslash \hat{N}$ according to $\mu$. For all other solvable roommate markets, $\hat{\varphi}$ assigns the set of stable matchings. Thus, core $\varsubsetneqq \hat{\varphi}$.

Proposition 4 On the domain of solvable roommate markets (and on any of its subdomains), solution $\hat{\varphi}$ (defined in Example 1) satisfies individual rationality, Pareto optimality, (weak) unanimity, mutually best, consistency, and weak resource sensitivity.

We prove Proposition 4 in Appendix A.3.

\subsection{Core characterizations}

Next, we strengthen the marriage market characterizations of the core presented in Theorem 1 (Toda's 2006, Theorem 3.2) and Theorem 2 in two ways. First, for marriage markets we replace the respective population monotonicity property with its corresponding population sensitivity property and second, we extend this characterization to the domains of no-odd-rings and of solvable roommate markets.

\section{Theorem 3 (Three Core Characterizations: Competition Sensitivity)}

(a) On the domain of marriage markets,

(b) On the domain of no-odd-rings roommate markets, a solution satisfies weak unanimity, weak competition sensitivity, and consistency if and only if it equals the core.

(c) On the domain of solvable roommate markets, a solution satisfies weak unanimity, competition sensitivity, and consistency if and only if it equals the core.

Proof Let $\varphi$ be a solution on any of the domains of Theorem 3. By Propositions 1, 2 , and 3 , the core satisfies weak unanimity, (weak) competition sensitivity, and consistency. Let $\varphi$ be weakly unanimous, competition sensitive, and consistent. Then, by Lemma 6, $\varphi$ is a subsolution of the core and by Lemma 1, $\varphi$ equals the core. We establish (a) and (b) with weak competition sensitivity instead of competition sensitivity by using Lemma 6 ' instead of Lemma 6.

Lemma 6(d) establishes a corresponding impossibility result to Theorem 3 on the domain of all roommate markets. Whether we can strengthen Theorem 3(c) by using weak competition sensitivity instead of competition sensitivity is an open problem.

With Example 2 in Appendix A. 2 we illustrate why Theorem 3 might not hold if the set of potential agents is finite. 
Theorem 4 (Three Core Characterizations: Resource Sensitivity)

(a) On the domain of marriage markets,

(b) On the domain of no-odd-rings roommate markets, a solution satisfies weak unanimity, weak resource sensitivity, and consistency if and only if it equals the core.

(c) On the domain of solvable roommate markets, a solution satisfies weak unanimity, resource sensitivity, and consistency if and only if it equals the core.

Proof Let $\varphi$ be a solution on any of the domains of Theorem 4. By Propositions 1, 2, and 3, the core satisfies weak unanimity, (weak) resource sensitivity, and consistency. Let $\varphi$ be weakly unanimous, resource sensitive, and consistent. Then, by Lemma 7, $\varphi$ is a subsolution of the core and by Lemma 1, $\varphi$ equals the core. We establish (a) and (b) with weak resource sensitivity instead of resource sensitivity by using Lemma $7^{\prime}$ instead of Lemma 7.

Lemma 7(d) establishes a corresponding impossibility result to Theorem 4 on the domain of all roommate markets. Solution $\hat{\varphi}$ (defined in Example 1) demonstrates that Theorem 4(c) for solvable roommate markets cannot be strengthened by using weak resource sensitivity instead of resource sensitivity.

Theorems 1 and 2 show that on the domain of classical marriage markets, the core is the unique solution satisfying weak unanimity, consistency, and own-side or otherside population monotonicity. Both results follow from our "population sensitivity characterizations of the core" for marriage markets (Theorems 3(a) and 4(a)).

Corollary 1 (Two Core Characterizations for Marriage Markets)

On the domain of marriage markets, a solution satisfies weak unanimity, consistency, and

(1) own-side population monotonicity,

(2) other-side population monotonicity,

if and only if it equals the core.

We prove Corollary 1 in Appendix B. An example constructed along the lines of Example 2 in Appendix A.2 illustrates why Corollary 1 (1) might not hold if the set of potential agents is finite.

We next show the independence of properties in Theorems 3 and 4 (these examples can also be used to show the independence of properties in Corollary 1).

The solution $\varphi^{s}$ on the domains in Theorems 3 and 4 that always assigns the matching at which all agents are single satisfies (weak) competition and (weak) resource sensitivity, consistency, but not weak unanimity.

On the domains in Theorems 3 and 4 any proper subsolution of the core satisfies (weak) unanimity, (weak) competition and (weak) resource sensitivity (Proposition 3), but not consistency (Lemma 1).

The Pareto solution $P O$ on the domains in Theorems 3 and 4 satisfies (weak) unanimity and consistency, but neither weak competition nor weak resource sensitivity (see Klaus 2011, Example 2). 


\section{Remark 4 (Pareto Optimality)}

Since Pareto optimality implies weak unanimity, we can use this stronger efficiency property in all of our results (the same solutions that establish the independence of properties in Theorems 3 and 4 can be used again).

\section{Conclusion}

In this paper, we make some positive contribution to the study of one-sided markets: a marriage market characterization (Toda's 2006, Theorem 3.2) is extended to various (solvable) roommate market domains (Theorem 3 with (weak) competition sensitivity) and some new complementary characterizations are established (Theorem 4 with (weak) resource sensitivity). Furthermore, corresponding impossibility results (Lemmas 6(d) and 7(d)) and corresponding marriage market results (Corollary 1) are obtained. At first sight, our paper thus parallels Klaus (2011) with the difference that it extends Toda's "consistency core characterization" (Toda's 2006, Theorem 3.2) instead of his "Maskin monotonicity core characterization" (Toda's 2006, Theorem 3.1). Establishing the results mentioned above validates the new population sensitivity properties introduced by Klaus (2011). However, there are some interesting and important differences between our paper and Klaus (2011) that go beyond the use of consistency instead of Maskin monotonicity. A new feature in our article is the addition of the no-odd-rings domain (in particular, single-peaked, single-dipped, and distance-based preference domains constitute relevant no-odd-rings roommate market domains). Maybe even more importantly, we had to develop a new proof strategy to extend previous marriage market results to roommate markets. We explain this latter aspect below.

All results in Klaus (2011) could essentially be established by following Toda's (2006, Theorem 3.1) proof steps. ${ }^{16}$ However, this is not true anymore when extending Toda's (2006, Theorem 3.2) second result. It is impossible to extend Toda's crucial Lemma 3.4 because one cannot show that a rule that satisfies (individual rationality,) mutually best and consistency must be a subset of the core-see our counterexample in Example 1. Hence, we have developed a new proof strategy for the original result (Toda's 2006, Theorem 3.2) that also works for the extension to roommate markets. ${ }^{17}$

An example of a result (or results) where a similar asymmetry of proof techniques can be observed between two-sided and one-sided markets are the random paths to stability result(s) by Roth and Van de Vate (1990) and by Diamantoudi et al. (2004).

\footnotetext{
16 Although adjusting those steps to work with the new population sensitivity properties did require a lot of work and technical skills.

17 Two-sided markets show a lot of regularity that is missing from roommate markets: one main feature often used in "marriage market proofs" is the polarization that occurs within the core; in particular, the existence of side-optimal stable matchings and the possibility to use side-monotonic (or side-greedy) arguments is key in many proofs for two-sided matching markets. This polarization essentially creates the marriage market core regularity used by Toda's (2006) when adding or removing agents. For roommate markets (i.e., in our paper), the absence of polarization and regularity (not so surprisingly) causes "trouble" in that the core basically can suddenly collapse or expand (in contrast, in marriage markets, the core lattice is only truncated or expanded on one of its "sides").
} 
We believe that the study of "one-sided (roommate) market techniques" also provides valuable insights for more general models such as coalition formation or network formation.

Acknowledgments B. Klaus thank the Netherlands Organisation for Scientific Research (NWO) for its support under Grant VIDI-452-06-013.

\section{Appendix A: Proofs of Lemmas 1, 6' ${ }^{\prime} 7^{\prime}$, and Proposition 4}

\section{A.1 Proof of Lemma 1}

Before proving Lemma 1, we state and prove a so-called Bracing Lemma (which is a typical consistency result for many economic models, see Thomson 2009).

\section{Lemma 8 (Bracing Lemma)}

(a) Let $(N, R)$ be a marriage market. For each $\mu \in \operatorname{core}(N, R)$, there exists a marriage market $\left(N^{\prime}, R^{\prime}\right)$ (see also Toda's 2006, Lemma 5.8),

(b) Let $(N, R)$ be a no-odd-rings roommate market. For each $\mu \in \operatorname{core}(N, R)$, there exists a no-odd-rings roommate market $\left(N^{\prime}, R^{\prime}\right)$,

(c) Let $(N, R)$ be a solvable roommate market. For each $\mu \in \operatorname{core}(N, R)$, there exists a solvable roommate market $\left(N^{\prime}, R^{\prime}\right)$, such that $N \subseteq N^{\prime}, R_{N}^{\prime}=R$, $\operatorname{core}\left(N^{\prime}, R^{\prime}\right)=\left\{\mu^{\prime}\right\}$, and $\mu_{N}^{\prime}=\mu$.

Proof For the proof of (a), let $(N, R) \in \mathfrak{D}_{M}$, for the proof of (b), let $(N, R) \in \mathfrak{D}_{N O R}$, and for the proof of $(\mathrm{c})$, let $(N, R) \in \mathfrak{D}_{S}$. If $|\operatorname{cor} e(N, R)|=1$, then there is nothing to prove. Let $\operatorname{core}(N, R)=\left\{\mu, \mu_{1}, \ldots, \mu_{k}\right\}$ for some $k \geq 1$. Since the core is Pareto optimal, there exists $i^{*} \in N$ such that $\mu\left(i^{*}\right) P_{i^{*}} \mu_{1}\left(i^{*}\right)$.

First, consider the extension $\left(N^{*}, R^{*}\right)$ of $(N, R)$ that is obtained by adding a newcomer $n^{*} \in \mathbb{N} \backslash N$ such that $N^{*}=N \cup\left\{n^{*}\right\}$ and $R^{*} \in R^{N^{*}}$ is such that

(i) $R_{N}^{*}=R$,

(ii) for all $i \in N \backslash\left\{i^{*}\right\}$ and all $j \in N$ (possibly $i=j$ ), $j P_{i}^{*} n^{*}$, i.e., for every agent in $N$-except agent $i^{*}$-agent $n^{*}$ is the least preferred agent,

(iii) $\mu\left(i^{*}\right) P_{i^{*}}^{*} n^{*} P_{i^{*}}^{*} \mu_{1}\left(i^{*}\right)$,

i.e., agent $i^{*}$ ranks the newcomer $n^{*}$ between agents $\mu\left(i^{*}\right)$ and $\mu_{1}\left(i^{*}\right)$, and

(iv) for all $j \in N \backslash\left\{i^{*}\right\}, i^{*} P_{n^{*}}^{*} n^{*} P_{n^{*}}^{*} j$, i.e., the newcomer finds only agent $i^{*}$ acceptable.

For the proof of (a), $\left(N^{*}, R^{*}\right)$ is a marriage market by choosing agent $n^{*}$ 's gender to be opposite of agent $i^{*}$ 's gender.

For the proof of (b), we note that $\left(N^{*}, R^{*}\right)$ is a no-odd-rings roommate market because the newcomer $n^{*}$ cannot be part of a ring given that $n^{*}$ finds only $i^{*}$ acceptable (by the definition of a ring, any element of a ring has at least two acceptable agents).

For the proof of (c), we show that $\left(N^{*}, R^{*}\right)$ is also a solvable roommate market. Note that by construction $\mu \cup\left\{n^{*}\right\} \in \operatorname{core}\left(N^{*}, R^{*}\right)$. Thus $\left(N^{*}, R^{*}\right)$ is solvable. 
Second, we prove that $\left(N^{*}, R^{*}\right)$ has fewer stable matchings than $(N, R)$. By construction, $\mu \cup\left\{n^{*}\right\} \in \operatorname{cor} e\left(N^{*}, R^{*}\right)$. Note that by the so-called Lone Wolf Theorem (e.g., Klaus and Klijn 2010, Theorem 1) any agent who is single in one stable matching is single in all other stable matchings. Thus, only matchings of the form $\mu^{*} \cup\left\{n^{*}\right\}$, $\mu^{*} \in \mathcal{M}(N, R)$, can be stable for roommate market $\left(N^{*}, R^{*}\right)$. Furthermore, since the core is consistent (Proposition 2), if for any $\tilde{\mu} \in \mathcal{M}(N, R), \tilde{\mu} \cup\left\{n^{*}\right\} \in \operatorname{core}\left(N^{*}, R^{*}\right)$, then $\tilde{\mu} \in \operatorname{core}(N, R)$. Hence, $\left|\operatorname{core}\left(N^{*}, R^{*}\right)\right| \leq|\operatorname{core}(N, R)|$. Finally, since $\left(i^{*}, n^{*}\right)$ blocks $\mu_{1} \cup\left\{n^{*}\right\}, \mu_{1} \cup\left\{n^{*}\right\} \notin \operatorname{core}\left(N^{*}, R^{*}\right)$. We conclude that $\left|\operatorname{core}\left(N^{*}, R^{*}\right)\right|<$ $|\operatorname{core}(N, R)|$ and $\mu \cup\left\{n^{*}\right\} \in \operatorname{core}\left(N^{*}, R^{*}\right)$.

Repeating this process of adding a newcomer to reduce the number of stable matchings at most $k$ times results in

(a) a marriage market $\left(N^{\prime}, R^{\prime}\right)$,

(b) a no-odd-rings roommate market $\left(N^{\prime}, R^{\prime}\right)$,

(c) a solvable roommate market $\left(N^{\prime}, R^{\prime}\right)$,

such that $N \subseteq N^{\prime}, R_{N}^{\prime}=R$, core $\left(N^{\prime}, R^{\prime}\right)=\left\{\mu^{\prime}\right\}$, and $\mu_{N}^{\prime}=\mu$.

The Bracing Lemma (Lemma 8) is a key element in the proof of Lemma 1

\section{Lemma 1}

(a) On the domain of marriage markets (see also Toda's 2006, Lemma 3.6),

(b) On the domain of no-odd-rings roommate markets,

(c) On the domain of solvable roommate markets,

no proper subsolution of the core satisfies consistency.

Proof Let $\varphi$ be a solution on any of the domains $\mathfrak{D}^{\prime}$ of Lemma 1 that is a consistent subsolution of the core. Let $(N, R) \in \mathfrak{D}^{\prime}$ and $\mu \in \operatorname{core}(N, R)$. Then, by the Bracing Lemma (Lemma 8), there exists a roommate market $\left(N^{*}, R^{*}\right) \in \mathfrak{D}^{\prime}$ with $\operatorname{core}\left(N^{*}, R^{*}\right)=\left\{\mu^{*}\right\}$ such that $(N, R)$ is a reduced market of $\left(N^{*}, R^{*}\right)$ at $\mu^{*}$ and $\mu_{N}^{*}=\mu$. Since $\varphi$ is a subsolution of the core, $\varphi\left(N^{*}, R^{*}\right)=\left\{\mu^{*}\right\}$. As $\varphi$ is consistent, $\mu \in \varphi(N, R)$. So, $\operatorname{cor} e(N, R) \subseteq \varphi(N, R)$. Since $\varphi$ is a subsolution of the core, $\varphi(N, R) \subseteq \operatorname{core}(N, R)$. Hence, $\varphi(N, R)=\operatorname{cor} e(N, R)$.

A. 2 Lemmas $6^{\prime}$ and $7^{\prime}$ and their Proofs

\section{Lemma $6^{\prime}$}

(a) On the domain of marriage markets,

(b) On the domain of no-odd-rings roommate markets,

if a solution satisfies weak unanimity, weak competition sensitivity, and consistency, then it is a subsolution of the core.

\section{Lemma $7^{\prime}$}

(a) On the domain of marriage markets,

(b) On the domain of no-odd-rings roommate markets, if a solution satisfies weak unanimity, weak resource sensitivity, and consistency then it is a subsolution of the core. 
Because the first parts of the proofs of the above lemmas are identical, we prove both lemmas together and indicate the steps when either weak competition sensitivity or weak resource sensitivity are used. Throughout the proof we will list partial information on agents' preferences. For instance, $j R_{i} k R_{i} l$ will be represented as $R_{i} \mid j k l$ in a partial preference table.

Proof Let $\mathfrak{D}^{\prime} \in\left\{\mathfrak{D}_{M}, \mathfrak{D}_{N O R}\right\}$. Assume that $\varphi$ satisfies weak unanimity, weak competition sensitivity [weak resource sensitivity], and consistency, but that it is not a subsolution of the core. Thus, there exists $(N, R) \in \mathfrak{D}^{\prime}$ and a matching $\mu \in \varphi(N, R)$ such that $\mu \notin \operatorname{core}(N, R)$. By Lemma 3 [Lemma 4](a) and (b), $\varphi$ satisfies mutually best. By Lemma 2(a) and (b), $\varphi$ satisfies individual rationality. Hence, there exists a blocking pair $\{i, j\}, i \neq j$, for $\mu$ such that $j P_{i} \mu(i) R_{i} i$ and $i P_{j} \mu(j) R_{j} j$. Let $N^{\prime}=\{i, j, \mu(i), \mu(j)\}$ and consider the reduced market $\left(N^{\prime}, R_{N^{\prime}}\right) \in \mathfrak{D}^{\prime}$ of $(N, R)$ at $\mu$. By consistency,

$$
\mu_{N^{\prime}} \in \varphi\left(N^{\prime}, R_{N^{\prime}}\right)
$$

We consider three cases depending on the cardinality of $N^{\prime}$.

Case $1\left(\left|\mathbf{N}^{\prime}\right|=2\right)$ : Consider the reduced market $\left(N^{\prime}, R_{N^{\prime}}\right)$ where $N^{\prime}=\{i, j\}$ (note that $\left.\left(N^{\prime}, R_{N^{\prime}}\right) \in \mathfrak{D}_{M}\right)$. Agents $i$ and $j$ are mutually best agents for $\left(N^{\prime}, R_{N^{\prime}}\right)$. However, at the reduced matching $\mu_{N^{\prime}}$ they are not mutually matched. Hence, (1) contradicts mutually best.

Case 2 ( $\left.\left|\mathbf{N}^{\prime}\right|=3\right)$ : Consider the reduced market $\left(N^{\prime}, R_{N^{\prime}}\right)$ where $N^{\prime}=\{i, j, \mu(i)\}$. It is without loss of generality that we assume that agent $j$ is single. By individual rationality and $\{i, j\}$ being a blocking pair for $\mu$, agents' preferences are as follows:

$$
\begin{array}{l|l}
R_{i} & j \mu(i) i \\
R_{\mu(i)} & i \mu(i) \\
R_{j} & i j
\end{array}
$$

Weak Competition Sensitivity Step $\left(\right.$ Lemma $\left.6^{\prime}\right)$. Assume that $\left(\hat{N}^{\prime}, \hat{R}^{\prime}\right), \hat{N}^{\prime}=N^{\prime} \cup\{n\}$, is an extension of $\left(N^{\prime}, R_{N^{\prime}}\right)$ such that agents $\mu(i)$ and $n$ are mutually best agents for $\left(\hat{N}^{\prime}, \hat{R}^{\prime}\right)$ and agent $\mu(i)$ is the only one that finds $n$ acceptable and $n$ finds only $\mu(i)$ acceptable [the newcomer and preferences can be chosen such that $\left(\hat{N}^{\prime}, R_{\hat{N}^{\prime}}\right)$ is also a marriage / no-odd-rings roommate market]. By mutually best, for all $\mu^{\prime} \in \varphi\left(\hat{N}^{\prime}, \hat{R}^{\prime}\right)$, $\mu^{\prime}(n)=\mu(i)$. By weak competition sensitivity, for $\mu_{N^{\prime}} \in \varphi\left(N^{\prime}, R_{N^{\prime}}\right)$ there exists $\hat{\mu}^{\prime} \in \varphi\left(\hat{N}^{\prime}, \hat{R}^{\prime}\right)$ such that agents $i$ and $j$ are not mutually matched, i.e., $\hat{\mu}^{\prime}(i) \neq j$ (if not, then agents $i$ and $j$ are newly matched at $\hat{\mu}^{\prime}$, but both are better off). Hence, $\hat{\mu}^{\prime} \in \varphi\left(\hat{N}^{\prime}, \hat{R}^{\prime}\right)$ is the matching that mutually best matches agents $\mu(i)$ and $n$ and agents $i$ and $j$ are single.

Thus, $\left(\{i, j\}, R_{\{i, j\}}\right)$ is a reduced market of $\left(\hat{N}^{\prime}, \hat{R}^{\prime}\right)$ at $\hat{\mu}^{\prime}$ to $\{i, j\}$. Note that $i$ and $j$ are mutually best agents at $\left(\{i, j\}, R_{\{i, j\}}\right)$ and both single at $\hat{\mu}_{\{i, j\}}^{\prime}$. By consistency, $\hat{\mu}_{\{i, j\}}^{\prime} \in \varphi\left(\{i, j\}, R_{\{i, j\}}\right)$, which contradicts mutually best.

Weak Resource Sensitivity Step (Lemma $\left.7^{\prime}\right)$. Consider the roommate market $(\{i, j\}$, $\left.R_{\{i, j\}}\right)$. There exists a unanimously best complete matching $\bar{\mu}$ for (marriage, 
no-odd-rings) roommate market $\left(\{i, j\}, R_{\{i, j\}}\right): \bar{\mu}$ matches agent $i$ with agent $j$. Hence, by weak unanimity, $\varphi\left(\{i, j\}, R_{\{i, j\}}\right)=\{\bar{\mu}\}$ and $\bar{\mu}(i)=j$.

Consider the extension $\left(N^{\prime}, R_{N^{\prime}}\right)$ of $\left(\{i, j\}, R_{\{i, j\}}\right)$ that is obtained by adding newcomer $\mu(i)$. Because $\mu(i) \neq j$ and $\bar{\mu}(i)=j$, by weak resource sensitivity, $\mu(i) P_{i}$ $\bar{\mu}(i)=j$ or $\mu(j) P_{j} \bar{\mu}(j)=i$. This contradicts that $\{i, j\}$ is a blocking pair for $\mu$.

Case $3\left(\left|\mathbf{N}^{\prime}\right|=4\right)$ : Consider the reduced market $\left(N^{\prime}, R_{N^{\prime}}\right)$ where $N^{\prime}=\{i, j, \mu(i)$, $\mu(j)\}$. By individual rationality and $\{i, j\}$ being a blocking pair for $\mu$, agents' preferences are as follows:

$$
\begin{array}{l|l}
R_{i} & j \mu(i) i \\
R_{j} & i \mu(j) j \\
R_{\mu(i)} & i \mu(i) \\
R_{\mu(j)} & j \mu(j)
\end{array}
$$

If agents $i$ and $j$ are mutually best agents for $\left(N^{\prime}, R_{N^{\prime}}\right)$, then (1) contradicts mutually best. If $\left(N^{\prime}, R_{N^{\prime}}\right)$ is a marriage market, then agents $j$ and $\mu(i)$ and agents $i$ and $\mu(j)$ have the same gender. But then, agents $i$ and $j$ are mutually best agents for $\left(N^{\prime}, R_{N^{\prime}}\right)$ that are not mutually matched at $\mu_{N^{\prime}}$; a contradiction. Hence, $\mathfrak{D}^{\prime}=\mathfrak{D}_{N O R}$ and agents $i$ and $j$ not being mutually best agents for $\left(N^{\prime}, R_{N^{\prime}}\right)$ implies $\mu(j) P_{i} j P_{i} \mu(i) P_{i} i$ or $\mu(i) P_{j} i P_{j} \mu(j) P_{j} j$. Without loss of generality we assume that $\mu(i) P_{j} i P_{j} \mu(j) P_{j} j$. Thus, agents' preferences can be further restricted to:

$$
\begin{array}{l|l}
R_{i} & j \mu(i) i \\
R_{j} & \mu(i) i \mu(j) j \\
R_{\mu(i)} & i \mu(i) \\
R_{\mu(j)} & j \mu(j)
\end{array}
$$

Weak Competition Sensitivity Step $\left(\right.$ Lemma $\left.6^{\prime}\right)$. Assume that $\left(\hat{N}^{\prime}, \hat{R}^{\prime}\right), \hat{N}^{\prime}=N^{\prime} \cup\{n\}$, is an extension of $\left(N^{\prime}, R_{N^{\prime}}\right)$ such that agents $\mu(j)$ and $n$ are mutually best agents for $\left(\hat{N}^{\prime}, \hat{R}^{\prime}\right)$ and agent $\mu(j)$ is the only one that finds $n$ acceptable and $n$ finds only $\mu(j)$ acceptable [the newcomer and preferences can be chosen such that $\left(N^{\prime}, R_{N^{\prime}}\right)$ is a noodd-rings roommate market]. By mutually best, for all $\mu^{\prime} \in \varphi\left(\hat{N}^{\prime}, \hat{R}^{\prime}\right), \mu^{\prime}(n)=\mu(j)$. By weak competition sensitivity, for $\mu_{N^{\prime}} \in \varphi\left(N^{\prime}, R_{N^{\prime}}\right)$ there exists $\hat{\mu}^{\prime} \in \varphi\left(\hat{N}^{\prime}, \hat{R}^{\prime}\right)$ such that agents $i$ and $j$ are not mutually matched, i.e., $\hat{\mu}^{\prime}(i) \neq j$ (if not, then agents $i$ and $j$ are newly matched at $\hat{\mu}^{\prime}$, but both are better off).

(*) If $j P_{\mu(i)} i P_{\mu(i)} \mu(i)$ and $\hat{\mu}^{\prime}(j)=\mu(i)$, then agents $j$ and $\mu(i)$ are newly matched and both better off; contradicting the choice of $\hat{\mu}^{\prime}$ to satisfy weak competition sensitivity.

Consider the reduced market $\left(\{i, j, \mu(i)\}, R_{\{i, j, \mu(i)\}}\right) \in \mathfrak{D}_{N O R}$ of $\left(\hat{N}^{\prime}, \hat{R}^{\prime}\right)$ at $\hat{\mu}^{\prime}$.

$$
\begin{array}{l|l}
R_{i} & j \mu(i) i \\
R_{j} & \mu(i) i j \\
R_{\mu(i)} & i \mu(i)
\end{array}
$$

By weak competition sensitivity and consistency, $\bar{\mu}^{\prime} \equiv \hat{\mu}_{\{i, j, \mu(i)\}}^{\prime} \in \varphi(\{i, j, \mu(i)\}$, $\left.R_{\{i, j, \mu(i)\}}\right)$ such that $\bar{\mu}^{\prime}(i) \neq j$. 
We continue with the joint proof of Lemmas $6^{\prime}$ and $7^{\prime}$ after establishing a corresponding proof step for weak resource sensitivity.

Weak Resource Sensitivity Step (Lemma $\left.7^{\prime}\right)$. Consider the reduced market $\left(\{i, j, \mu(i)\}, R_{\{i, j, \mu(i)\}}\right) \in \mathfrak{D}_{N O R}$ obtained from agent $\mu(j)$ leaving $\left(N^{\prime}, R_{N^{\prime}}\right)$.

$$
\begin{array}{l|l}
R_{i} & j \mu(i) i \\
R_{j} & \mu(i) i j \\
R_{\mu(i)} & i \mu(i)
\end{array}
$$

By weak resource sensitivity, for $\mu_{N^{\prime}} \in \varphi\left(N^{\prime}, R_{N^{\prime}}\right)$ there exists $\bar{\mu}^{\prime} \in \varphi(\{i, j, \mu(i)\}$, $\left.R_{\{i, j, \mu(i)\}}\right)$ such that agents $i$ and $j$ are not mutually matched, i.e., $\bar{\mu}^{\prime}(i) \neq j$ (if not, then agents $i$ and $j$ are not mutually matched at $\mu_{N^{\prime}}$ anymore, but both are worse off).

(*) If $j P_{\mu(i)} i P_{\mu(i)} \mu(i)$ and $\hat{\mu}^{\prime}(j)=\mu(i)$, then agents $j$ and $\mu(i)$ are not mutually matched at $\mu_{N^{\prime}}$ anymore and both are worse off; contradicting the choice of $\hat{\mu}^{\prime}$ to satisfy weak resource sensitivity.

We now finish the proof of Lemmas $6^{\prime}$ and $7^{\prime}$ with a joint step. In the previous steps we have established the existence of

$$
\bar{\mu}^{\prime} \in \varphi\left(\{i, j, \mu(i)\}, R_{\{i, j, \mu(i)\}}\right) \text { such that } \bar{\mu}^{\prime}(i) \neq j \text {. }
$$

If $\bar{\mu}^{\prime}(i)=\mu(i)$, then $\{i, j\}$ is a blocking pair for $\bar{\mu}^{\prime}$. If $\bar{\mu}^{\prime}(i)=i$ and $\bar{\mu}^{\prime}(j)=j$, then $\{i, j\}$ is a blocking pair for $\bar{\mu}^{\prime}$. The remaining case to discuss is $\bar{\mu}^{\prime}(i)=i$ and $\bar{\mu}^{\prime}(j)=\mu(i)$. If $i P_{\mu(i)} j P_{\mu(i)} \mu(i)$, then $\{i, j, \mu(i)\}$ constitutes an odd ring; contradicting $\left(\{i, j, \mu(i)\}, R_{\{i, j, \mu(i)\}}\right) \in \mathfrak{D}_{N O R}$. By $(*), j P_{\mu(i)} i P_{\mu(i)} \mu(i)$ is not possible. Hence, $i P_{\mu(i)} \mu(i) P_{\mu(i)} j$ and $\bar{\mu}^{\prime}(j)=\mu(i)$. However, $\bar{\mu}^{\prime}(j)=\mu(i)$ violates individual rationality, a contradiction.

To summarize, we either obtain a contradiction, or blocking pair $\{i, j\}$ for $\bar{\mu}^{\prime} \in$ $\varphi\left(\{i, j, \mu(i)\}, R_{\{i, j, \mu(i)\}}\right)$. Since $\left|\left\{i, j, \bar{\mu}^{\prime}(i), \bar{\mu}^{\prime}(j)\right\}\right| \leq 3$, Cases 1 and 2 now imply a contradiction.

The following three-agent example demonstrates why Lemmas 3, 6, 6', and Theorem 3 might not hold if the set of potential agents is finite. Note that the simple idea of Example 2 (namely to add a non-core matching to all roommate markets containing the finite set of potential agents) can be extended to any finite set of potential agents (if the set of potential agents is even, then one should only add the additional matching for roommate markets without a unanimously best complete matching).

Example 2 Assume that the set of potential agents is $\{1,2,3\}$ and denote by $\mu^{12}$ the matching where agents 1 and 2 are matched. Then, for all roommate markets $(N, R) \in \mathfrak{D}^{\prime} \subseteq \mathfrak{D}_{S}$,

$$
\tilde{\varphi}(N, R)= \begin{cases}\operatorname{core}(N, R) \cup\left\{\mu^{12}\right\} & \text { if }|N|=3 \text { and } \mu^{12} \in I R(N, R), \\ \operatorname{core}(N, R) & \text { otherwise. }\end{cases}
$$

It is easy to check that $\tilde{\varphi}$ satisfies weak unanimity, (weak) competition sensitivity, and consistency, but it is not a subsolution of the core. 
A. 3 Proof of Proposition 4

Proposition 4 On the domain of solvable roommate markets (and on any of its subdomains), solution $\hat{\varphi}$ (defined in Example 1) satisfies individual rationality, Pareto optimality, (weak) unanimity, mutually best, consistency, and weak resource sensitivity.

Proof We prove Proposition 4 for $\mathfrak{D}^{\prime}=\mathfrak{D}_{S}$. It is easy to see that solution $\hat{\varphi}$ (defined in Example 1) satisfies individual rationality, Pareto optimality, (weak) unanimity, and mutually best.

We partition the domain of solvable roommate markets $\mathfrak{D}_{S}$ into the subdomain $\mathfrak{D}_{S S}$ of solvable roommate markets with the separable submarket $(\hat{N}, \hat{R})$ and its complement solvable domain $\mathfrak{D}_{C S}=\mathfrak{D}_{S} \backslash \mathfrak{D}_{S S}$ (without separable submarket $(\hat{N}, \hat{R}))$.

Note that for roommate markets with a separable submarket $\left(\mathfrak{D}_{S S}\right)$, the set of agents $\hat{N}$ constitutes an even ring of size 4 . Hence removing an agent from this submarket dissolves the solvability of the market due to an odd ring at the top of preferences of 3 agents in $\hat{N}$. Therefore, it is not possible to obtain an extension $\left(N^{\prime}, R^{\prime}\right) \in \mathfrak{D}_{S S}$ of $(N, R) \in \mathfrak{D}_{C S}$ by adding a newcomer $n \in \mathbb{N} \backslash N$. Furthermore, since each agent in $\hat{N}$ finds only agents in $\hat{N}$ acceptable, for all extensions $\left(N^{\prime}, R^{\prime}\right) \in \mathfrak{D}_{S}$ of $(N, R) \in \mathfrak{D}_{S S}$ that are obtained by adding a newcomer $n \in \mathbb{N} \backslash N$, and for all $\mu^{\prime} \in \hat{\varphi}\left(N^{\prime}, R^{\prime}\right)$, $\mu^{\prime}(\hat{N})=\hat{N}$.

Weak Resource Sensitivity. In order to show that $\hat{\varphi}$ is weakly resource sensitive, let $(N, R) \in \mathfrak{D}_{S}$ and consider the extension $\left(N^{\prime}, R^{\prime}\right) \in \mathfrak{D}_{S}$ of $(N, R)$ obtained by adding a newcomer $n \in \mathbb{N} \backslash N$.

Case 1. Let $(N, R) \in \mathfrak{D}_{C S}$. It follows that $\left(N^{\prime}, R^{\prime}\right) \in \mathfrak{D}_{C S}$. By construction of $\hat{\varphi}$, $\hat{\varphi}(N, R)=\operatorname{core}(N, R)$ and $\hat{\varphi}\left(N^{\prime}, R^{\prime}\right)=\operatorname{core}\left(N^{\prime}, R^{\prime}\right)$. By Proposition 3, the core is weakly resource sensitive. Hence, for all $\mu^{\prime} \in \operatorname{cor} e\left(N^{\prime}, R^{\prime}\right)=\hat{\varphi}\left(N^{\prime}, R^{\prime}\right)$ there exists $\mu \in \operatorname{core}(N, R)=\hat{\varphi}(N, R)$ such that for all $i, j \in N$ [possibly $i=j$ ] that are not mutually matched at $\mu^{\prime}$ anymore, at least one is better off.

Case 2. Let $(N, R) \in \mathfrak{D}_{S S}$ and assume $\left(N^{\prime}, R^{\prime}\right) \in \mathfrak{D}_{C S}$. By construction of $\hat{\varphi}$, $\hat{\varphi}(N, R) \supsetneq \operatorname{core}(N, R)$ and $\hat{\varphi}\left(N^{\prime}, R^{\prime}\right)=\operatorname{core}\left(N^{\prime}, R^{\prime}\right)$. By Proposition 3 , the core is weakly resource sensitive. Hence, for all $\mu^{\prime} \in \operatorname{core}\left(N^{\prime}, R^{\prime}\right)=\hat{\varphi}\left(N^{\prime}, R^{\prime}\right)$ there exists $\mu \in \operatorname{core}(N, R) \subsetneq \hat{\varphi}(N, R)$ such that for all $i, j \in N$ [possibly $i=j$ ] that are not mutually matched at $\mu^{\prime}$ anymore, at least one is better off.

Case 3. Let $(N, R) \in \mathfrak{D}_{S S}$ and assume $\left(N^{\prime}, R^{\prime}\right) \in \mathfrak{D}_{S S}$. Since each agent in $\hat{N}$ finds only agents in $\hat{N}$ acceptable, for all $\mu \in \hat{\varphi}(N, R), \mu(\hat{N})=\hat{N}$, and for all $\mu^{\prime} \in \hat{\varphi}\left(N^{\prime}, R^{\prime}\right), \mu^{\prime}(\hat{N})=\hat{N}$. Therefore, we treat the set of agents $\hat{N}$ separately from the set of agents $N \backslash \hat{N}$.

For agents in $\hat{N}$ : Note that in both roommate markets $(N, R)$ and $\left(N^{\prime}, R^{\prime}\right), \hat{\varphi}$ matches agents in $\hat{N}$ according to $\hat{\mu}, \hat{\mu}^{\prime}$, or $\hat{\mu}^{\prime \prime}$. Therefore, for all $\mu^{\prime} \in \hat{\varphi}\left(N^{\prime}, R^{\prime}\right)$ there exists $\mu \in \hat{\varphi}(N, R)$ such that $\mu_{\hat{N}}^{\prime}=\mu_{\hat{N}} \in\left\{\hat{\mu}, \hat{\mu}^{\prime}, \hat{\mu}^{\prime \prime}\right\}$. In particular, $\mu \in \hat{\varphi}(N, R)$ is such 
that for all $i, j \in \hat{N}$ [possibly $i=j$ ] that are not mutually matched at $\mu^{\prime}$ anymore, at least one is better off.

For agents in $N \backslash \hat{N}$ : Note that in both roommate markets $(N, R)$ and $\left(N^{\prime}, R^{\prime}\right), \hat{\varphi}$ matches agents in $N \backslash \hat{N}$ (respectively $\left.N^{\prime} \backslash \hat{N}\right)$ according to $\hat{\varphi}\left(N \backslash \hat{N}, R_{N \backslash \hat{N}}\right)=$ $\operatorname{core}\left(N \backslash \hat{N}, R_{N \backslash \hat{N}}\right)$ (respectively $\left.\hat{\varphi}\left(N^{\prime} \backslash \hat{N}, R_{N^{\prime} \backslash \hat{N}}\right)=\operatorname{core}\left(N^{\prime} \backslash \hat{N}, R_{N^{\prime} \backslash \hat{N}}\right)\right)$. By Proposition 3, the core is weakly resource sensitive. Hence, for all $\mu^{\prime} \in \hat{\varphi}\left(N^{\prime}, R^{\prime}\right)$, $\mu_{N^{\prime} \backslash \hat{N}}^{\prime} \in \operatorname{cor} e\left(N^{\prime} \backslash \hat{N}, R_{N^{\prime} \backslash \hat{N}}\right)$ and there exists $\mu_{N \backslash \hat{N}} \in \operatorname{core}\left(N \backslash \hat{N}, R_{N \backslash \hat{N}}\right)$ such that for all $i, j \in N \backslash \hat{N}$ [possibly $i=j$ ] that are not mutually matched at $\mu_{N^{\prime} \backslash \hat{N}}^{\prime}$ with each other anymore, at least one is better off. In particular, we can choose $\mu \in \hat{\varphi}(N, R)$ such that $\mu_{\hat{N}}^{\prime}=\mu_{\hat{N}}$.

Cases 1-3 imply that for all $(N, R),\left(N^{\prime}, R^{\prime}\right) \in \mathfrak{D}_{S}$ such that $\left(N^{\prime}, R^{\prime}\right)$ is an extension of $(N, R)$ obtained by adding a newcomer $n \in \mathbb{N} \backslash N$ and for all $\mu^{\prime} \in \hat{\varphi}\left(N^{\prime}, R^{\prime}\right)$ there exists $\mu \in \hat{\varphi}(N, R)$ such that for all $i, j \in N$ [possibly $i=j$ ] that are not mutually matched at $\mu^{\prime}$ anymore, at least one is better off.

Consistency. In order to show that $\hat{\varphi}$ is consistent, let $(N, R) \in \mathfrak{D}_{S}, \mu \in \hat{\varphi}(N, R)$, and assume that $\left(N^{\prime}, R_{N^{\prime}}\right) \in \mathfrak{D}_{S}$ is a reduced market of $(N, R)$ at $\mu$ to $N^{\prime}{ }^{18}$

Case 1. Let $(N, R) \in \mathfrak{D}_{C S}$. By construction of $\hat{\varphi}, \hat{\varphi}(N, R)=\operatorname{cor} e(N, R)$ and $\operatorname{core}\left(N^{\prime}, R^{\prime}\right) \subseteq \hat{\varphi}\left(N^{\prime}, R^{\prime}\right)$. By Proposition 2, the core is consistent. Hence, for all $\mu \in \operatorname{core}(N, R)=\hat{\varphi}(N, R), \mu_{N^{\prime}} \in \operatorname{core}\left(N^{\prime}, R^{\prime}\right) \subseteq \hat{\varphi}(N, R)$.

Case 2. Let $(N, R) \in \mathfrak{D}_{S S}$. By construction of $\hat{\varphi}, \mu=\left(\mu^{*}, \tilde{\mu}\right)$ for some $\mu^{*}=\mu_{N \backslash \hat{N}} \in$ $\operatorname{core}\left(N \backslash \hat{N}, R_{N \backslash \hat{N}}\right)$ and for some $\tilde{\mu}=\mu_{\hat{N}} \in\left\{\hat{\mu}, \hat{\mu}^{\prime}, \hat{\mu}^{\prime \prime}\right\}$.

Case 2.1. Let $\left(N^{\prime}, R^{\prime}\right) \in \mathfrak{D}_{S S}$. Thus, $\hat{N} \subseteq N^{\prime}$ and $\mu_{N^{\prime}}=\left(\mu_{N^{\prime} \backslash \hat{N}}^{*}, \tilde{\mu}\right)$. By Proposition 2, the core is consistent. Hence, $\mu_{N^{\prime} \backslash \hat{N}}^{*} \in \operatorname{core}\left(N^{\prime} \backslash \hat{N}, R_{N^{\prime} \backslash \hat{N}}\right)$. Thus, by construction of $\hat{\varphi}, \mu_{N^{\prime}}=\left(\mu_{N^{\prime} \backslash \hat{N}}^{*}, \tilde{\mu}\right) \in \hat{\varphi}\left(N^{\prime}, R^{\prime}\right)$.

Case 2.2. Let $\left(N^{\prime}, R^{\prime}\right) \in \mathfrak{D}_{C S}$. By construction of $\hat{\varphi}, \hat{\varphi}\left(N^{\prime}, R^{\prime}\right)=\operatorname{cor} e\left(N^{\prime}, R^{\prime}\right)$. By Proposition 2, the core is consistent. Hence, since $\mu^{*} \in \operatorname{core}\left(N \backslash \hat{N}, R_{N \backslash \hat{N}}\right), \mu_{N^{\prime} \backslash \hat{N}}^{*} \in$ $\operatorname{core}\left(N^{\prime} \backslash \hat{N}, R_{N^{\prime} \backslash \hat{N}}\right)$. Since $\left(N^{\prime}, R^{\prime}\right) \in \mathfrak{D}_{C S}$, either $\hat{N} \cap N^{\prime}=\emptyset$ or $\left|\hat{N} \cap N^{\prime}\right|=2$. If $\hat{N} \cap N^{\prime}=\emptyset$, then $N^{\prime} \backslash \hat{N}=N^{\prime}$, which implies $\mu_{N^{\prime}}=\mu_{N^{\prime} \backslash \hat{N}}^{*} \in \operatorname{core}\left(N^{\prime}, R^{\prime}\right)=$ $\hat{\varphi}\left(N^{\prime}, R^{\prime}\right)$. Assume that $\left|\hat{N} \cap N^{\prime}\right|=2$ and $\hat{N} \cap N^{\prime}=\{i, j\}$. Since the reduced market $\left(N^{\prime}, R^{\prime}\right)$ is obtained from $(N, R) \in \mathfrak{D}_{S S}, \tilde{\mu}(i)=j$. Furthermore, agents $i$ and $j$ are mutually best agents for $\left(N^{\prime}, R^{\prime}\right)$. Hence, for all $\mu^{\prime} \in \hat{\varphi}\left(N^{\prime}, R^{\prime}\right)=\operatorname{core}\left(N^{\prime}, R^{\prime}\right)$, $\mu^{\prime}(i)=j$. Thus, by construction of $\hat{\varphi}, \mu_{N^{\prime}}=\left(\mu_{N^{\prime} \backslash \hat{N}}^{*}, \tilde{\mu}_{\{i, j\}}\right) \in \operatorname{core}\left(N^{\prime}, R^{\prime}\right)=$ $\hat{\varphi}\left(N^{\prime}, R^{\prime}\right)$.

\footnotetext{
18 Note that solvability is closed under reduction for $\hat{\varphi}$, i.e., for all $(N, R) \in \mathfrak{D}_{S}$ and for all matchings $\mu \in \hat{\varphi}(N, R)$, the reduced market $\left(N^{\prime}, R^{\prime}\right)$ of $(N, R)$ at $\mu$ is also solvable.
} 


\section{Appendix B: Consistency and population monotonicity for (classical) marriage markets}

A classical marriage market (Gale and Shapley 1962) $(N, R)$ is such that $N$ is the union of two disjoint sets $M$ and $W$ and each agent in $M$ (respectively $W$ ) has restricted preferences over being matched to agents in the set $W$ (respectively $M$ ) and being single (instead of having preferences over $N$ ). Furthermore, matching agents of the same gender is not feasible (instead of matching agents of the same gender being individually irrational). Definitions and results labeled "on the domain of (classical) marriage markets" in this appendix apply to the classical marriage market domain as well as to our "marriage-roommate market" domain. We first introduce two population monotonicity properties.

Own-side population monotonicity (simply called population monotonicity by Toda's 2006) states that if additional men (women) enter the market, then all incumbent men (women) are weakly worse off. We formalize a somewhat weaker version of own-side population monotonicity by restricting population changes to one newcomer at a time (this "weak own-side population monotonicity" implies the original own-side population monotonicity by adding men (women) one by one).

Own-Side Population Monotonicity for Marriage Markets: A solution $\varphi$ on the domain of (classical) marriage markets is own-side population monotonic if the following holds. Let $(N, R)$ be a marriage market and assume that $\left(N^{\prime}, R^{\prime}\right), N^{\prime}=N \cup\{n\}$, is an extension of $(N, R)$ and the newcomer $n$ is a man [woman]. Then, for all $\mu \in$ $\varphi(N, R)$ there exists $\mu^{\prime} \in \varphi\left(N^{\prime}, R^{\prime}\right)$ such that for all men $m \in N, \mu(m) R_{m} \mu^{\prime}(m)$ [for all women $w \in N, \mu(w) R_{w} \mu^{\prime}(w)$ ].

Other-side population monotonicity states that if additional men (women) enter the market, then all incumbent women (men) are weakly better off. We formalize a somewhat weaker version of other-side population monotonicity by restricting population changes to one newcomer at a time (this "weak other-side population monotonicity" implies the original other-side population monotonicity by adding men (women) one by one).

Other-Side Population Monotonicity for Marriage Markets: A solution $\varphi$ on the domain of (classical) marriage markets is other-side population monotonic if the following holds. Let $(N, R)$ be a marriage market and assume that $\left(N^{\prime}, R^{\prime}\right), N^{\prime}=N \cup\{n\}$, is an extension of $(N, R)$ and the newcomer $n$ is a man [woman]. Then, for all $\mu^{\prime} \in$ $\varphi\left(N^{\prime}, R^{\prime}\right)$ there exists $\mu \in \varphi(N, R)$ such that for all women $w \in N, \mu^{\prime}(w) R_{w} \mu(w)$ [for all men $m \in N, \mu^{\prime}(m) R_{m} \mu(m)$ ].

Proposition 5 On the domain of (classical) marriage markets, the core satisfies ownside and other-side population monotonicity.

Proof By Toda's (2006, Proposition 2.1) the core satisfies own-side population monotonicity for the domain of classical marriage markets. However, we show both properties in a symmetric way using results by Crawford (1991). Note that the classical marriage market results used in this proof also hold for "marriage-roommate" markets (see Remark 1). 
Let $(N, R),\left(N^{\prime}, R^{\prime}\right)$ be such that $\left(N^{\prime}, R^{\prime}\right)$ is an extension of $(N, R)$ with $N^{\prime}=N \cup\{n\}$, without loss of generality newcomer $n$ being a man. We denote the men-optimal stable matching and the women-optimal stable matching (Gale and Shapley 1962) by $\mu_{M}, \mu_{W}$ for roommate market $(N, R)$ and $\mu_{M}^{\prime}, \mu_{W}^{\prime}$ for roommate market $\left(N^{\prime}, R^{\prime}\right)$.

By Roth and Sotomayor (1990, Corollary 2.14), for all $\mu \in \operatorname{core}(N, R)$ and all men $m \in N, \mu R_{m} \mu_{W}$. By Crawford (1991, Theorem 1), for all men $m \in N, \mu_{W} R_{m} \mu_{W}^{\prime}$. Hence for all $\mu \in \operatorname{cor} e(N, R)$, there exists $\mu^{\prime} \in \operatorname{cor} e\left(N^{\prime}, R^{\prime}\right)$, namely $\mu^{\prime} \equiv \mu_{W}^{\prime}$, such that for all men $m \in N, \mu R_{m} \mu^{\prime}$. This implies own-side population monotonicity.

By Roth and Sotomayor (1990, Corollary 2.14), for all $\mu^{\prime} \in \operatorname{core}\left(N^{\prime}, R^{\prime}\right)$ and all women $w \in N, \mu^{\prime} R_{w} \mu_{M}^{\prime}$. By Crawford (1991, Theorem 2), for all women $w \in N$, $\mu_{M}^{\prime} R_{w} \mu_{M}$. Hence for all $\mu^{\prime} \in \operatorname{core}\left(N^{\prime}, R^{\prime}\right)$, there exists $\mu \in \operatorname{cor} e(N, R)$, namely $\mu \equiv \mu_{M}$, such that for all women $w \in N, \mu^{\prime} R_{w} \mu$. This implies other-side population monotonicity.

We next establish some relations between properties for marriage markets.

Lemma 9 On the domain of (classical) marriage markets, other-side population monotonicity, and consistency imply individual rationality.

Proof Assume that $\varphi$ satisfies weak unanimity and consistency, but not individual rationality. Then, there exists a marriage market $(N, R)$, a matching $\mu \in \varphi(N, R)$, and without loss of generality a man $m \in N$, such that $m P_{m} \mu(m)$ (alternatively we could assume that there exists a woman $w \in N$ such that $\left.w P_{w} \mu(w)\right)$.

Let $N^{\prime}=\{m, \mu(m)\}$. Then, marriage market $\left(N^{\prime}, R_{N^{\prime}}\right)$ is a reduced market of $(N, R)$ at $\mu$ and by consistency, $\mu_{N^{\prime}} \in \varphi\left(N^{\prime}, R_{N^{\prime}}\right)$ and $\mu_{N^{\prime}}(m)=\mu(m)$.

Let $\bar{N}=\{m\}$. Marriage market $\left(\bar{N}, R_{\bar{N}}\right)$ is a one agent market with only one possible matching. Hence, $\varphi\left(\bar{N}, R_{\bar{N}}\right)=\{\bar{\mu}\}$ with $\bar{\mu}(m)=m$. Furthermore, $\left(N^{\prime}, R^{\prime}\right)$ is an extension of $\left(\bar{N}, R_{\bar{N}}\right)$ and the newcomer $\mu(m)$ is a woman. Thus, by other-side population monotonicity, for all $\mu^{\prime} \in \varphi\left(N^{\prime}, R^{\prime}\right), \mu^{\prime}(m) R_{m} \bar{\mu}(m)=m$. This contradicts $\mu_{N^{\prime}} \in \varphi\left(N^{\prime}, R_{N^{\prime}}\right)$ and $m P_{m} \mu_{N^{\prime}}(m)$.

Klaus (2011) shows that on the domain of marriage markets, weak resource sensitivity is essentially a weaker property than other-side population monotonicity (individual rationality is added to ensure that no two agents of the same gender are matched, see Remark 1).

Lemma 10 (Klaus 2011, Lemma 2) On the domain of (classical) marriage markets, individual rationality and other-side population monotonicity imply weak resource sensitivity.

Strictly speaking Klaus (2011) does not give the proof for classical marriage markets, but the proof of Lemma 10 is essentially the same as Klaus (2011, Lemma 2).

The following two lemmas by Toda's (2006) are used in the proof of Theorem 2.

Lemma 5 (Toda's 2006, Lemma 3.4). On the domain of classical marriage markets, if a solution $\varphi$ satisfies individual rationality, mutually best, and consistency, then it is a subsolution of the core. 
Lemma 11 (Toda's 2006, Lemma 3.6) On the domain of classical marriage markets, no proper subsolution of the core satisfies consistency.

Next, we restate and prove Theorem ??.

Theorem 2 On the domain of classical marriage markets, a solution satisfies weak unanimity, other-side population monotonicity, and consistency if and only if it equals the core.

Proof The core satisfies weak unanimity and consistency Toda's (2006). By Proposition 5 , the core is other-side population monotonic.

Let $\varphi$ satisfy weak unanimity, other-side population monotonicity, and consistency. Then, by Lemma 9, $\varphi$ is individually rational. Thus, by Lemma 10, $\varphi$ satisfies weak resource sensitivity. Hence, by Lemma 4(a) (the proof essentially remains the same on the domain of classical marriage markets), $\varphi$ satisfies mutually best. Thus, by Lemma $5, \varphi$ is a subsolution of the core. Then, Lemma 11 implies that $\varphi$ equals the core.

We conclude this appendix by restating and proving Corollary 1. For completeness, we first state the following lemma.

Lemma 12 (Klaus 2011, Lemma 1) On the domain of marriage markets, individual rationality and own-side population monotonicity imply weak competition sensitivity.

Corollary 1 (Two Characterizations of the Core for Marriage Markets)

On the domain of marriage markets, a solution satisfies weak unanimity, consistency, and

(1) own-side population monotonicity;

(2) other-side population monotonicity;

if and only if it equals the core.

Proof Let $\varphi$ be a solution on the domain of marriage markets. Let $\varphi$ be weakly unanimous, consistent, and (1) own-side population monotonic or (2) other-side population monotonic. By Lemma 9, $\varphi$ is individually rational. Thus, (1) by Lemma 12, $\varphi$ is weakly competition sensitive and (2) by Lemma $10, \varphi$ is weakly resource sensitive. Hence, (1) by Theorem 3(a), $\varphi$ equals the core and (2) by Theorem 4(a), $\varphi$ equals the core.

\section{References}

Chung KS (2000) On the existence of stable roommate matchings. Games Econ Behav 33:206-230

Crawford VP (1991) Comparative statics in matching markets. J Econ Theory 54:389-400

Demange G, Wooders M (eds) (2004) Group formation in economics; networks, clubs and coalitions. Cambridge University Press, Cambridge

Diamantoudi E, Miyagawa E, Xue L (2004) Random paths to stability in the roommate problem. Games Econ Behav 48:18-28

Gale D, Shapley LS (1962) College admissions and the stability of marriage. Am Math Mon 69:9-15

Jackson MO (2008) Social and economic networks. Princeton University Press, Princeton 
Klaus B (2008) Competition and resource sensitivity in marriage and roommate markets. Harvard Business School Working Paper 09-072. http://ideas.repec.org/p/hbs/wpaper/09-072.html

Klaus B (2011) Competition and resource sensitivity in marriage and roommate markets. Games Econ Behav 72:172-186

Klaus B, Klijn F (2010) Smith and Rawls share a room: stability and medians. Soc Choice Welf 35:647-667

Özkal-Sanver I (2010) Impossibilities in roommates problems. Math Soc Sci 59:360-363

Roth AE, Sotomayor MAO (1990) Two-sided matching: a study in game-theoretic modeling and analysis. Cambridge University Press, Cambridge

Roth AE, Van de Vate JH (1990) Random paths to stability in two-sided matching. Econometrica 58: 1475-1480

Sasaki H, Toda M (1992) Consistency and characterization of the core of two-sided matching problems. J Econ Theory 56:218-227

Thomson W (2009) Consistent allocation rules. Monograph. Forthcoming

Toda M (2006) Monotonicity and consistency in matching markets. Int J Game Theory 34:13-31 